\title{
À PROPOS DU LIBRO DEI SOGNI DE FEDERICO FELLINI. CRASH ET DEUIL SUR LA PAGE. UN CURIEUX PROBLÈME DE DATATION
}

CHRISTIAN GAILLARD

PHANÊS • VOLUME $3 \cdot 2020$ • PP. 116-162

https://doi.org/10.32724/phanes.2020.Gaillard 


\begin{abstract}
Tout au long de sa vie, Fellini s'est plu à dessiner à tout propos et en tous lieux. En 1970, son analyste, le Dr Bernhard, l'a invité à dessiner et à écrire ses rêves. Ce qu'il a fait bien au-delà de la fin de cette analyse, en 1975.Après de longues disputes entre ses héritiers, ces dessins et écrits, qu'il avait réunis sous le titre Il Libro dei sogni, ont été publiés par la Fondazione Fellini, de Rimini, qui a organisé un congrès à ce propos en 2007. L'essai qu'on va lire s'arrête à deux dessins du Maestro, qui ne se trouvent pas dans ce Libro, et qui ne sont pas datés. D'où une enquête qui s'appuie sur une série d'indices et de repères pour tenter de dater ces deux dessins, aujourd'hui conservés à la Fondation Fellini pour le cinéma, à Sion, en Suisse. Cette enquête conduit à une réflexion qui porte sur la thématique et la dynamique internes de l'œuvre et de la vie de l'artiste, et plus généralement sur celles de la création.
\end{abstract}

\title{
KEY WORDS
}

Fellini, Libro dei sogni, Dr Bernhard, Jung, Livre Rouge. 


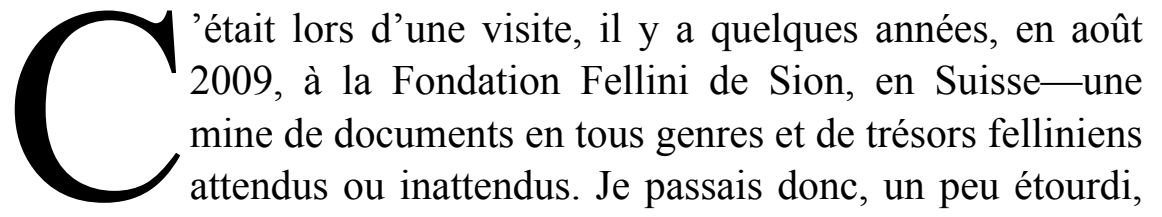
ébloui, d'une photographie de plateau à une affiche des années 1950, d'une lettre autographe à un souvenir de tournage, m'arrêtant ici ou là devant un objet ayant appartenu ou servi au Maestro, lorsque tout soudain, que vois-je? Deux dessins de Fellini, alors inédits, que me fait découvrir Stéphane Marti, le maître de ces lieux. Deux scènes, vivement colorées et solidement organisées, aussi impressionnantes l'une que l'autre par leur tenue formelle et par l'intensité de leur dramaturgie. De quoi tomber en arrêt (ill. 1 et 2).

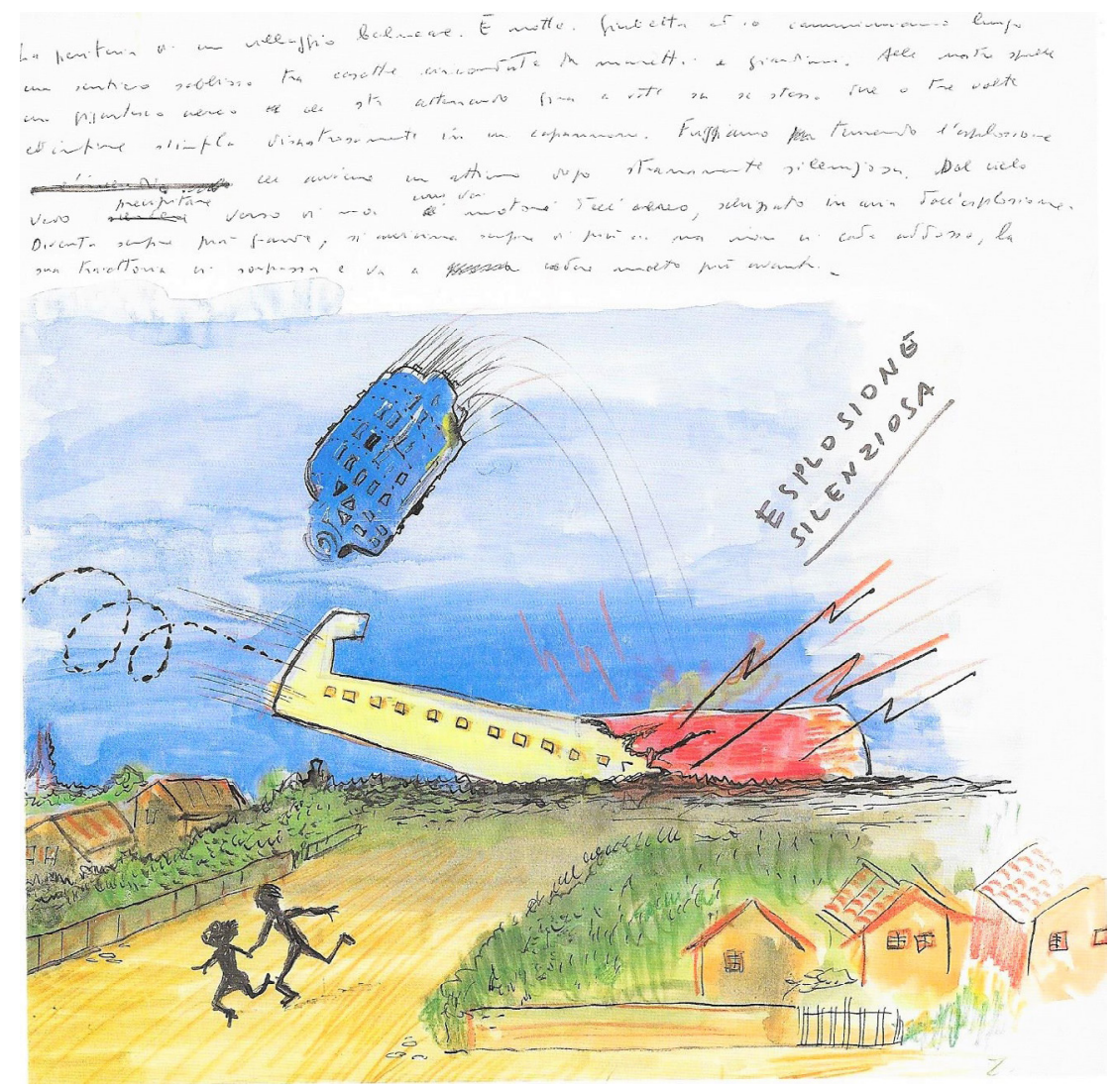

Ill. 1 (32,5 cm x $32 \mathrm{~cm})$. Courtesy Fondation Fellini pour le cinéma, Sion. 


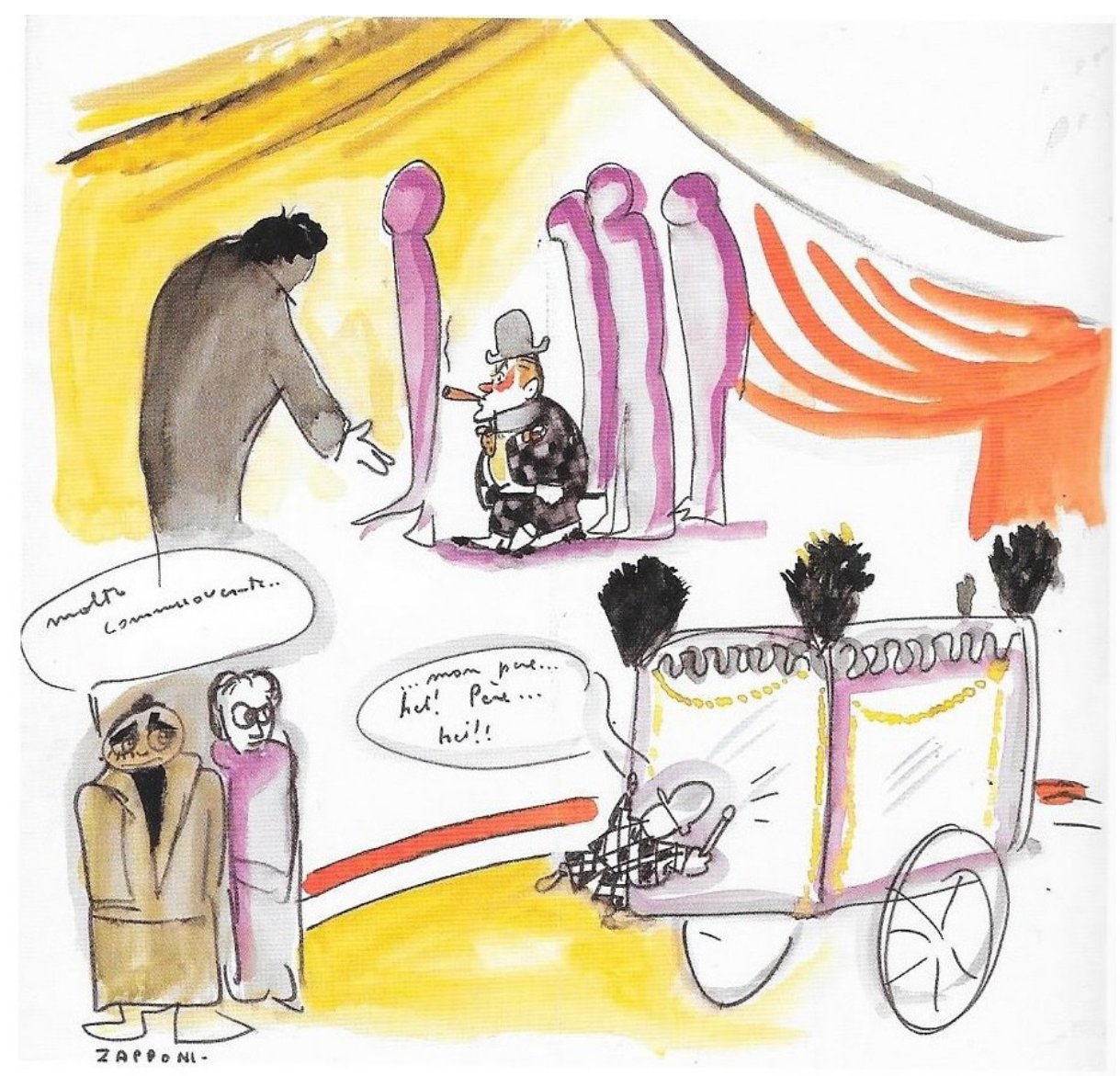

Ill. $2(34,5 \mathrm{~cm}$ x $32 \mathrm{~cm})$. Courtesy Fondation Fellini pour le cinéma, Sion.

Sur l'un, c'est, tout manifestement, le crash d'un avion. Sur l'autre, tout aussi manifestement, ce sont les pleurs d'un clown accablé par un deuil, ou feignant de l'être. Comment voir ces dessins, ces deux scènes ? Et comment apprécier leur place dans l'histoire et dans la dynamique de l'œuvre de Fellini, et aussi peut-être, sait-on jamais, de la vie du cinéaste ? ${ }^{1}$

\footnotetext{
${ }^{1}$ Que mon lecteur ne s'effraye pas de cet apparent engagement de mon propos dans la psychobiographie. J'ai eu l'occasion de dire à plus d'une reprise de quel œil je regarde cette orientation, si fréquentée, de la psychanalyse de l'art. On verra bientôt, au fil de ces pages, quel tour prendra une possible considération des rapports entre la dynamique d'une ouvre et le cours d'une vie.
} 


\section{UNE APPROCHE ET ACCROCHE OBSTINÉE}

Je suis d'autant plus impressionné en découvrant ces dessins que l'œuvre cinématographique de Fellini m'avait déjà sérieusement mobilisé, et de longue date. En fait depuis ma découverte aussi surprise qu'infiniment admirative de sa Dolce Vita au tout début des années 60, alors que j'étais encore tout jeune étudiant aux universités de Lausanne et de Genève ${ }^{2}$ une découverte nourrie ensuite de bien de façons, et surtout étrangement renouvelée lorsque, bien des années plus tard, devenu psychanalyste à Paris, j'ai reçu dans mon cabinet la visite inattendue du cinéaste canadien Damian Pettigrew, qui apportait alors la dernière main à la réalisation pour Arte de son excellent film Fellini, Je suis un grand menteur. ${ }^{3}$ C'était en 2002. Damian Pettigrew, qui travaillait à ce film, voulait mon avis d'analyste sur quelques dessins, alors inédits, du Maestro, et de là sur ses rêves.

Mais comment donner un quelconque avis sur les rêves en questionet de là sur Fellini! - en n'ayant sous les yeux que les quelques pages éparses qu'il me montrait alors ? J'avais refusé, bien sûr, mais non sans regret, de me lancer dans un exercice aussi périlleux, qui, face à ces pages, me laissait d'ailleurs perplexe. ${ }^{4}$

Et voilà que quelques années plus tard, la Fondazione Fellini de Rimini m'annonce la parution prochaine - si longtemps attendue, discutée et disputée-du Libro dei sogni de Fellini, et l'organisation, à cette occasion, d'un congrès qui devait se tenir, évidemment, à Rimini, pour saluer cette publication et frayer des voies de recherche à ce propos. Pour me convaincre de participer à l'événement et d'y apporter mon regard d'analyste, d'analyste jungien, sur ce livre, les organisateurs de ce congrès m'en envoient un pré-tirage, dans une présentation encore provisoire, d'un format bien différent de celui de la publication à venir, mais enfin complète, ou censément complète, et conforme à la composition et à la

\footnotetext{
${ }^{2}$ La vie universitaire en ces lieux était animée notamment par les projections organisées par Freddy Buache à la Cinémathèque de Lausanne.

${ }^{3}$ De ce réalisateur, on connaissait notamment ses films sur Beckett et sur Balthus. On lui doit aussi un livre d'entretiens avec Fellini, publié sous le même titre par L'Arche à Paris, en 1994, avec d'intéressants croquis de Fellini (Fellini, Pettigrew 1994).

${ }^{4}$ Dans les conditions d'une psychanalyse, c'est bien sûr le patient qui fait l'essentiel du travail — c'est pourquoi, depuis Lacan, on ne le dit plus 'patient', mais 'analysant'. Quant à l'analyste, il accompagne, et parfois intervient, pour relever quelque fait, détail ou thème négligé, ou soutenir, par amplification, certaines de ses associations ou certains de ses questionnements (cf Gaillard 2001).
} 
présentation chronologique du livre en cours de publication. De quoi me donner enfin quelque prise sur les dessins et les notes de Fellini à propos de ses rêves. Je ne pouvais que me risquer à m'y plonger, dans ce livre de rêves, avec l'espoir de ne pas m'y perdre. J'acceptai cette invitation.

Ce congrès s'est tenu à Rimini les 9 et 10 novembre 2007. Il était présidé par Tullio Kezich, un proche de Fellini et un de ses meilleurs biographes $^{5}$. Et les congressistes invités à y présenter une communication en sont repartis avec sous le bras-si l'on peut dire-l'énorme, très encombrant et très pesant Librone du cinéaste dans sa première et toute fraîche édition italienne, par Rizzoli.

Je reprends ici à mon compte ce terme très italien de 'Librone'-ce qu'on peut traduire, en somme, par 'Gros Bouquin'-, car on le doit à Fellini lui-même. C'est ainsi qu'il se plaisait à désigner l'ouvrage, un bien gros livre, en effet, composé à l'origine de feuillets de différents formats, qu'il gardait plus ou moins caché dans un tiroir - un tiroir, il est vrai, pas toujours fermé à clef-, et dont il pouvait aussi donner quelques pages ici ou là à certains de ses proches ${ }^{6}$. D'où la présentation assez complexe de ce Livre qui dans sa première édition italienne comporte plus de 400 pages, et rassemble en fait trois parties distinctes.

La première partie de ce Livre comporte 244 pages, et couvre la période comprise entre le 30 novembre 1960 et le 2 août 1968. La seconde en compte 154. Elle commence en février 1973 et se termine en 1982, avec donc, notons-le, car il nous faudra revenir sur cette remarque, tout un temps sans rêves entre l'une et l'autre. À quoi s'ajoute une troisième partie composée de feuillets épars dessinés et écrits à différents moments de la vie de l'auteur, jusqu'en 1990. Avec une quatrième partie, plus brève, où se trouvent publiées des 'Feuilles offertes'. En fin de volume, les éditions italienne et française de ce livre présentent une transcription typographique des propos manuscrits de Fellini, qui sont traduits en français dans les

\footnotetext{
${ }^{5}$ Les Actes de ce congrès ont été édités sous le titre Federico Fellini. Il Libro dei miei sogni (2007). Ma communication s'intitulait 'Dire et non-dit du rêve' et a été publiée en français et en anglais. Puis en italien sous le titre L'Inconscio creatore (2009), dans un livre écrit avec Lella Ravasi Bellochio qui a aussi présenté une communication au congrès de Rimini. Jean A. Gili, qui est un de nos meilleurs historiens et critiques de cinéma, notamment du cinéma italien, a publié ensuite son Fellini. Le Magicien du réel (2009), avec une iconographie particulièrement riche et parlante. Sam Stourdzé, qui a aussi participé à ce congrès, a publié cette même année 2009 le catalogue de son exposition Fellini, au Jeu de Paume à Paris, sous le titre Fellini. La Grande Parade, avec là aussi une iconographie remarquablement riche et parlante, ainsi qu'un numéro spécial de Beaux Arts Magazine intitulé Tutto Fellini (2009b) auquel j'ai contribué.

${ }^{6}$ Comment ne pas penser ici à cet autre 'Librone' qu'est le Livre Rouge de Jung ? Ils sont côte-à-côte dans ma bibliothèque. J'y reviendrai.
} 
éditions de Flammarion. Ces rêves sont le plus souvent datés par Fellini en haut de leur représentation dessinée et des propos manuscrits qui les accompagnent. Ce n'est pas le cas des deux dessins de Sion. L'ensemble porte ainsi sur près de trente ans de rêves dessinés et écrits de la main de l'auteur, de novembre 1960 à août 1990, dont les quatre ans de son analyse avec le Dr Bernhard.

On sait en effet l'intérêt de Fellini pour la psychanalyse et son attention à ce qui peut se découvrir, se dire et se vivre là. Ses biographes nous rapportent comment après une première expérience assez malheureuse avec l'analyste freudien Emilio Servadio, il s'est engagé dans une analyse apparemment très suivie avec le fondateur du mouvement jungien en Italie, le Dr Ernst Bernhard. Cette analyse durera des derniers mois de 1960 à l'été de 1965 . $^{7}$

Flammarion a publié la même année 2007 une édition française de ce Livre. Cette édition française se présente avec la même pagination que l'édition italienne de Rizzoli, ce qui permettra à notre lecteur de se référer aussi bien à l'une qu'à l'autre de ces deux éditions lorsque j'évoquerai telle ou telle de ses pages. Sauf que l'édition française se présente dans une dimension sensiblement plus réduite, de $25 \mathrm{~cm}$ sur 33, ce qui a pour effet de réduire la reproduction de toute une partie des dessins et des propos autographes de Fellini - et on verra que le format des dessins de Sion qui vont nous occuper seront une des données à prendre en compte pour leur datation. ${ }^{8}$

En cette année 2020, à l'occasion des 100 ans de la naissance du Maestro et des nombreuses publications et émissions de radio ou de télévision qui ont accompagné la célébration de cet événement, cet éditeur français vient de publier une nouvelle édition de son livre, bien sûr épuisé, de 2007. Cette édition est toute semblable à la première, y compris pour ce qui est de sa pagination, ce qui permettra à mon lecteur de s'y reporter aussi s'il ne dispose pas de la première publication de cet éditeur ou de celle de Rizzoli, qui est celle dont je me sers depuis le congrès de Rimini.

\footnotetext{
${ }^{7}$ Une analyse qui s'est déroulée, bien sûr, sur un mode assez particulier du fait de la personnalité singulière du Dr Bernhard, de celle de Fellini, et du fait aussi de ce moment de l'histoire de la psychanalyse, en particulier en Italie. J'ai discuté cette analyse à partir de nos pratiques cliniques d'aujourd'hui dans mon article des Cahiers Jungiens de Psychanalyse (2009), qui reprend le titre de ma communication présentée au congrès de Rimini et la développe.

${ }^{8} \mathrm{Au}$ lecteur et regardeur de ces dessins, je recommande de se reporter à l'édition italienne de Rizzoli-même si l'édition française de Flammarion offre une traduction française des propos de Fellini qui accompagnent souvent le dessin des scènes de ses rêves, ce qui peut en faciliter l'accès.
} 
D'où, aujourd'hui, ces pages où je reprends, complète, et surtout relance et développe autrement une série d'essais que j'ai été amené à publier après ce congrès. ${ }^{9}$

Pour tenter de mieux faire place à ce qui se présente et de mieux appréhender ce qui se joue sur l'un et l'autre des dessins de Sion en établissant à quel moment et dans quel contexte de l'œuvre et aussi de la vie de Fellini ils ont été réalisés, voyons ce que peuvent nous apprendre le format, la tenue formelle et la thématique de chacun d'eux.En commençant par le premier de ces dessins, qu'on pourrait intituler, de loin, à première vue, Le crash de l'avion, ou Il y a du désastre dans l'air (ill.1).

\section{LE FORMAT, LA TENUE FORMELLE, ET LA THÉMATIQUE DE CETTE SCÈNE}

Ce dessin mesure $32,5 \mathrm{~cm}$ sur $32 \mathrm{~cm}$.

Or la première partie de ce Libro réunit des dessins d'un format sensiblement différent, de $34 \mathrm{~cm}$ sur $24 \mathrm{~cm}$, qui datent de novembre 1960 à août 1968, tandis que sa deuxième partie réunit des dessins de plus grandes dimensions, de $48 \mathrm{~cm}$ sur $34 \mathrm{~cm}$, qui datent de juin 1970 à juillet 1990, et que sa troisième partie, dite des 'Feuilles volantes' ('Fogli sparsi'), réunit des dessins de formats divers, qui datent juin 1970 à septembre $1981^{10}$. Il paraît donc assez probable, en première approximation, que ce dessin de crash de l'avion a été réalisé après 1968 et avant 1982. Après 1968, c'est-à-dire après la fin de l'analyse de Fellini avec le Dr Bernhard, ce qui pourrait bien nous intéresser, et après aussi la réalisation de Otto e mezzo (1963) et de Giulietta degli spiriti (1965) Et avant 1982, c'est-à-dire avant E la nave va (1983), Intervista (1987) et La voce della luna (1990). ${ }^{11}$

Voyons maintenant la question de la datation de cette page en considérant la tenue formelle de la scène qui s'y trouve représentée. On remarque que cette scène est organisée autour d'un double événement, un avion qui s'écrase au sol et explose, et la fuite éperdue de deux petits personnages qui s'enfuient à toutes jambes vers le bas gauche de la page.

\footnotetext{
${ }^{9}$ On les trouvera mentionnés au fil de ces notes.

${ }^{10}$ La quatrième partie de ce Livre, celle des 'Feuilles offertes', réunit des dessins qui datent d'octobre 1961 à juin 1981, mais ils sont aussi de plus grandes dimensions que celles du dessin de Sion.

${ }^{11}$ Gérald Morin, qui a été longtemps proche de Fellini et son assistant nous fait revisiter dans son film documentaire Sur les traces de Fellini (2013), l'élaboration et la réalisation de Roma (1972), de Amarcord (1973), et de Casanova (1976) dans leur contexte romain des années 1970.
} 
Le crash de l'avion et ce mouvement de fuite ont en commun d'être l'un et l'autre dessinés et mis en scène sur un mode remarquablement vif, presque violent, et par là fortement dramatisé. Or on peut observer qu'à partir de décembre 1961, les pages de ce Libro se trouvent progressivement plus agitées, et même bousculées, jusqu'à s'en trouver parfois presque désorganisées, et même éclatées, tandis qu'on voit et qu'on lit sur la page l'expression de sensations assez désagréables qui en viennent à menacer sérieusement l'équilibre des scènes données à voir. ${ }^{12}$ Ces sensations désagréables et dérangeantes étaient jusqu'alors tenues soigneusement en respect par le bon ordre de la représentation et la présentation plus soignée des propos écrits par le rêveur.

Dans les scènes que le rêveur dessine jusqu'au début de 1962 et dans l'écriture qui les accompagne, le personnage qui le représente reste généralement prudent. Il se tient à distance, ou il se met à distance, de ce qui progressivement se présente et pourrait bien le troubler sérieusement. Ainsi dans un rêve du 8 janvier 1962 se voit-il, ou le voit-on, perché sur des échasses, comme pour mieux se préserver de ce qui lui arrive de plus dérangeant, et de plus indistinct, de plus indifférencié, à partir des contrebas de lui-même (ill. 3). ${ }^{13}$

Il se trouve que c'est précisément quelques mois après le début de son analyse avec le Dr Bernhard en automne 1960 que se manifestent progressivement dans ses dessins les changements formels que je viens de signaler, tout se passant comme si Federico Fellini commençait alors à se montrer, à se trouver, moins sûr de ce qui se présente dans ses rêves. Et moins sûr de lui-même. Jusqu'à se sentir menacé par ce qui se produit alors dans ses rêves, et de là dans ses dessins et sous sa plume, et dans ses rapports à lui-même. Et après la fin de son analyse, une analyse interrompue, comme on sait, par la mort de son analyste, le 19 juin 1965, ce livre change plus encore d'allure.

Ses dessins se font alors plus complexes encore, plus touffus, plus sombres aussi. Et surtout plus éclatés. Ce qui correspondant à la mise en page d'une thématique qu'il nous convient maintenant d'observer, toujours pour tenter de situer ce dessin du crash de l'avion dans l'histoire des créations cinématographiques et, pour autant que faire se peut, et très prudemment, bien sûr, à pas comptés, dans celle de la vie de notre Maestro.

\footnotetext{
$\overline{12}$ J'ai développé cette observation dans mes articles de 2009.

${ }^{13}$ A la différence de Fellini dans son Libro, Jung, dans son Livre Rouge, apporte un soin très calligraphique — évidemment évolutif, mais constant—à la relation écrite de ses rencontres et découvertes visionnaires, ainsi qu'à la réalisation des dessins et peintures qui les accompagnent.
} 


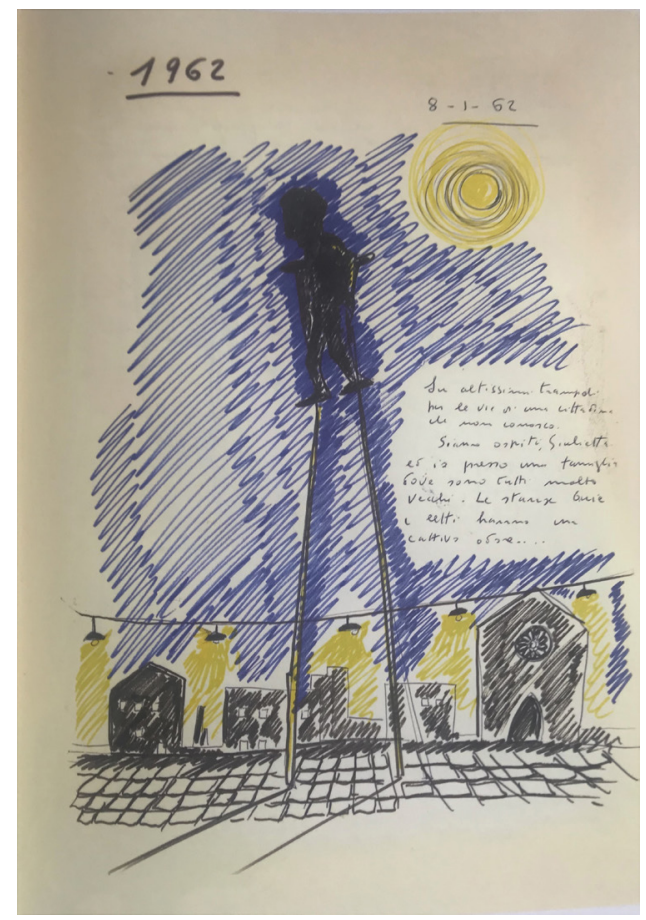

Ill. 3. Page 105 du Libro. Courtesy Fondazione Fellini, Rimini.

Dans ce dessin de Sion, on voit en effet un accident. Le brutal accident d'un avion qui s'écrase sur le sol et éclate dans une 'esplosione silenziosa'/ 'explosion silencieuse'. Ces mots sont écrits sur la page, et cet oxymore lui-même brutal rend l'événement plus impressionnant et inquiétant encore.

Un accident est toujours une catastrophe vécue, ou du moins entrevue, ou toute proche, un désastre qui menace, de fait, ou qu'on peut craindre. Surtout un accident d'avion. Or on ne peut qu'être frappé par la récurrence et l'insistance des chocs et entrechocs qui se multiplient dans ce Livre. Fellini vit peu à peu et relate des rêves et cauchemars affreux qu'il dessine d'une page à l'autre de son 'Librone'. Des tours s'effondrent, un train s'enfonce dans la nuit, une voiture se révèle impossible à conduire, et un avion manque de s'écraser dans un tunnel (des pages 173 à 263, de février 1966 à la fin de la première partie du Libro en août 1968). Voilà qui vient de nous faire passer, sans trop de transition, subrepticement, de l'observation de la tenue formelle de ces pages à une attention qui de là se porte maintenant sur la thématique des rêves qui s'y trouvent dessinés 
et racontés. Ce qui bouscule le bon ordre de mon propos, qui voulait que nous considérions successivement le format, puis la tenue formelle, et enfin la thématique des pages de ce Livre. Ce bon ordre en sera d'autant plus bousculé que l'événement aéronautique qui nous occupe se reproduit à bien des reprises au fil des pages de ce Livre.

Dans une longue et plus qu'inquiétante série d'explosions inattendues et autres catastrophes semblables parfois évitées de justesse, des aéroplanes, qui pourtant paraissaient heureux de voler, se trouvent en sérieuse difficulté (pages 68, 174, 192, 205, 249, 287, 301, 343, 344, $364,400)$. Cette série se ramasse et se noue plus dramatiquement encore dans un rêve du 30 décembre 1980, publié dans la deuxième partie de ce Livre, où un dirigeable est non seulement menacé, mais s'avère aussi plus menaçant que jamais (ill. 4).

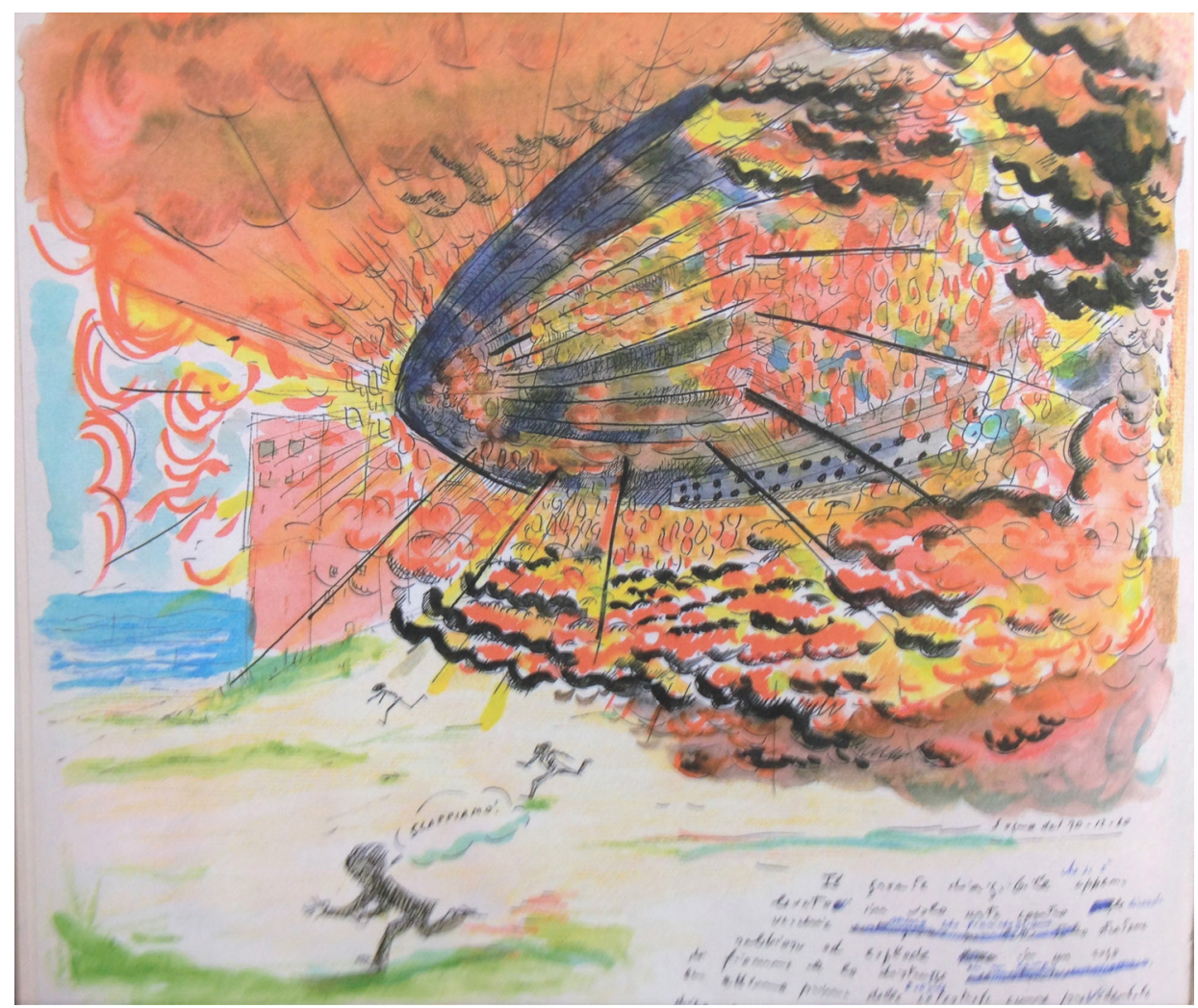

Ill. 4. Page 386 du Libro. Courtesy Fondazione Fellini, Rimini.

Dans ce rêve, ou plutôt dans ce dessin ${ }^{14}$, le rêveur se montre luimême gravement en danger. On le voit, paniqué, qui s'enfuit à toutes

\footnotetext{
${ }^{14}$ Il n'y a évidemment pas de texte du rêve. Chacun, au réveil, transcrit en mots, parfois aussi en images, ce qu'il a vécu dans la nuit.
} 
jambes. Comme sur la page conservée à Sion. Dans le dessin de cette scène, on peut lire, de la main de Fellini, 'Scappiamo!'/ 'Fuyons!'. ${ }^{15}$ Cette exclamation ne se trouve pas dans le dessin de Sion. Le texte manuscrit qui accompagne cette scène de décembre 1980 parle de 'catastrophe totale' et d' 'immense malheur', et aussi de 'signes prémonitoires' qu'il avait ou aurait pu percevoir à l'occasion de rêves récents, mais, remarquons le, sans que pour autant Fellini évoque la longue série des rêves précédents que nous venons de signaler, et qui, pour le premier d'entre eux, à la page 68 de son Libro dei sogni, date de juin 1961.

Quand il dessine et écrit ce rêve, Fellini note pourtant que dans cette scène il était vraiment plus jeune, presque un enfant - une remarque qui renvoie au passé, à son passé d'enfant, ou d'adolescent, ce qui inscrit l'événement dans une temporalité longue qui suscite son attention puisqu'il le note, mais, curieusement, sans pour autant qu'il s'y attarde, et sans non plus qu'apparemment il porte attention à la récurrence de ce type d'événements dans ses rêves, une récurrence et une insistance dont témoigne pourtant ce Livre qu'il dessine et écrit de sa main, et qui lui importe. Cette scène de l'aéronef qui explose est donc sérieusement récurrente. Ne la perdons pas de vue. Car, à l'évidence, ce Livre le montre, elle relève d'un scénario qui a obstinément occupé, et à répétition, notre rêveur ${ }^{16}$. On est tenté de dire qu'elle l'a sérieusement hanté, ou obsédé, qu'il ait voulu le voir ou non. ${ }^{17}$

\section{LES AÉRONEFS EXPLOSENT ET IL FAUT COMPRENDRE}

Ce rêve, ou plus exactement donc ce scénario, est manifestement si marquant pour notre rêveur et dessinateur qu'il est pratiquement le seul qu'on retrouvera ensuite sur de nombreuses autres pages de ce Livre. Mais dans un bien curieux désordre-si, du moins, on se rend attentif à l'écart entre sa pagination et les dates où il se retrouve. On le retrouvera en effet à la page 400 , toujours dans la deuxième partie de ce Livre, daté cette

\footnotetext{
${ }^{15}$ La traduction française de Flammarion donne 'Echappons-nous', mais à mon avis, ce verbe est un peu faible. Je propose donc plutôt: 'Fuyons!'.

${ }^{16} \mathrm{~J}$ 'ai exposé cette distinction entre scène et scénario dans un article intitulé 'L'Originaire est quotidien' (2016).

${ }^{17}$ Je présume que Fellini ne connaissait pas Charles Mauron. Il aurait peut-être prêté attention aux Métaphores obsédantes et mythe personnel (1963) de cet homme de lettres qui était son contemporain, et qui savait entendre ce qu'il lisait. Un autre auteur, qui lui était plus contemporain encore, aurait pu également l'intéresser, Gaston Bachelard, notamment par son L'Air et les songes (1941).
} 
fois du 25 février 1982, soit un peu plus de deux ans après celui que nous venons de voir. Dans le texte manuscrit qui accompagne le dessin et la relation de ce rêve, Fellini remarque que dans cette nouvelle scène du même drame, comme dans le rêve de 1980, il était encore enfant, sans pour autant que, dans ce Livre, il s'attarde à cette remarque, pas plus qu'il ne l'avait fait précédemment dans le rêve de juin 1961, lorsque il était déjà question de lui enfant.

Mais voilà que maintenant on peut lire sous sa plume cette sorte réflexion à la fois ferme et pensive, qui pourrait bien être une sorte d'interprétation: 'Ho pensato che quel sogno volevaannunciarmi l'inevitabile fine d'un vecchio modo di volare' / 'J'ai pensé que ce rêve voulait m'annoncer l'inévitable fin d'une manière de voler'. Ce qui le conduit à écrire ces mots impressionnants, et puissamment impératifs, qu'il souligne: 'Bisogna cambiare paesaggio, e modo, sistema, mezzo di viaggiare nelle proprie fantasie' / 'Il faut changer de paysage, de manière, de système, de moyen de transport, dans mes propres fantaisies' ${ }^{18}$

Et au-dessous de cette nouvelle scène de l'aéronef qui éclate, il dessine un sous-marin très noir et puissamment menaçant ${ }^{19}$. 'Tutto era nerissimo' / 'Tout était très noir', souligne-t-il. Et dans une note qui pourrait bien être postérieure à sa relation de ce rêve, il écrit encore, à propos de l'eau où se trouve ce sous-marin: 'E così buia perchè è ancora sconosciuta, appartienne all'inconscio' / 'Elle est si noire, car elle est encore inconnue, elle appartient à l'inconscient'. ${ }^{20}$

De plus, sur la même page, il écrit encore que dans un autre rêve de la même époque il découvrait des journaux publiant des photographies de lui-même avec ces légendes: '...scomparso, il re del cinema' / '...il a disparu, le roi du cinéma', '....sicuramente debellato, Fellini' / '... il est vaincu à coup sûr, Fellini'. ${ }^{21}$ C'est impressionnant. D'autant plus impressionnant qu'il ajoute sans ambages, cette fois sous la forme d'un commentaire qui lui serait venu après coup: 'Insomma, ero morto'/ 'Bref, j'étais mort'. Cette fois les mots qu'il écrit ne sont plus descriptifs. Fellini ici parle à la première personne: 'eromorto'. De quoi tomber en arrêt.

\footnotetext{
${ }^{18}$ Fantaisies? On pourrait aussi traduire ce 'fantasie' par fantasmes.

${ }^{19}$ Ce sous-marin est assez parent de celui qui se retrouvera en 1983 dans E la nave va. Il se retrouve aussi, de plus près, dans la page suivante du Libro (Fellini 2007: 401) avec en -dessous, un homme volant.

${ }^{20}$ Il est assez rare, remarquons-le aussi, que Fellini, dans ce Livre utilise ce terme d'inconscient (cf. Risset 2007).

21 'Debellato' est un terme très fort. Il dit en somme, si on veut bien l'entendre, que la guerre est perdue — en tous cas qu'une guerre est perdue.
} 
Et cinquante pages plus loin, à la page 451, on retrouve encore la relation, cette fois dans les pages volantes de ce Livre, de la même scène de crash et de fuite, avec le même 'Scappiamo !' / 'Fuyons!'. Mais, soulignons-le, ce rêve est antérieur de plus de deux ans à celui qui vient de nous arrêter, puisqu'il est daté du 30 décembre 1980. Sur la relation de ce rêve, on peut lire, toujours de la main de Fellini, en lettres majuscules, ces mots qui sonnent comme une injonction, ou du moins comme une invitation plus que pressante: 'Bisognerebbe riuscire a capire cos'è che si distrugge' / 'Il faudrait réussir à comprendre ce qui est détruit'.$^{22}$ Ce rêve s'avère pour lui si pressant, et si obsédant, que neuf mois plus tard, le 16 septembre 1981, il y reviendra et reprendra sa plume, pour ajouter, au bas de la même page, en lettres tout aussi majuscules, et plus grasses encore, cette interpellation apparemment, ou décidément, sans échappatoire: 'Cretino, non l'hai ancora capito ?' / 'Crétin, tu ne l'as pas encore compris ?' (ill. 5).

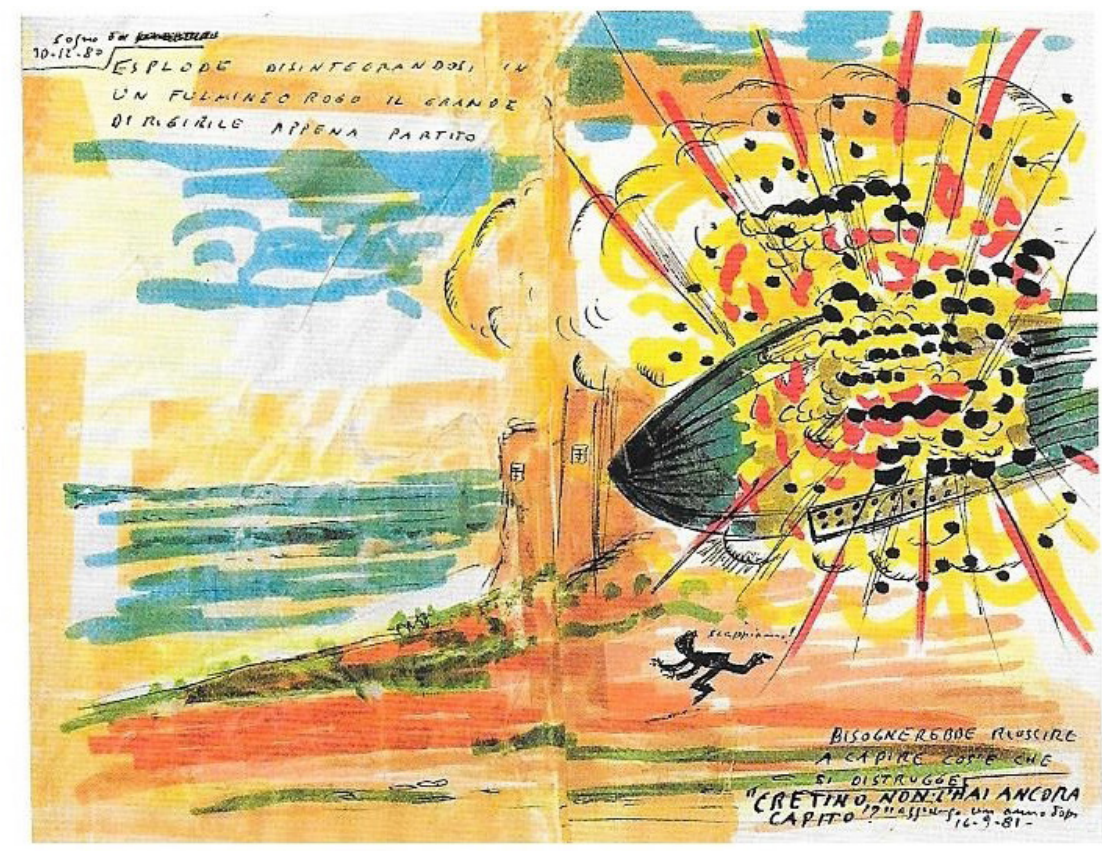

Ill. 5. Page 451 du Libro. Courtesy Fondazione Fellini, Rimini.

Comprendre quoi ? Revoyons ces pages. Et relisons ce qu'en s'est dit Fellini. Pour tenter de mieux voir, de mieux entendre, ce qui est signifié là-ou plutôt ce que Fellini, assez manifestement, tente de se signifier à lui-même.

${ }^{22}$ La traduction française de Flammarion donne ici, curieusement 'ce qui nous détruit', alors que le texte italien dit vraiment 'ce qui est détruit', ou 'ce qui se détruit'. 
La tâche n'est pas simple.

\section{L'ART DE BROUILLER LES PISTES}

La tâche n'est pas simple, car, on l'aura remarqué, si le premier de ces rêves se trouve à la page 386 de ce Livre et est daté du 30 décembre 1980, et si le deuxième, qui se trouve à la page 400, est daté du 25 février 1982, le troisième, qu'on trouve plus loin, à la page 451, est daté, lui, du 10 janvier 1980, et est donc antérieur aux deux autres. De plus, Fellini reviendra sur cette page, pour y ajouter, le 16 septembre 1981, donc après coup, l'exclamation où il s'apostrophe en se traitant de 'Cretino'.

La composition de ce Libro dei sogni, et de là, sa pagination, ne correspondent pas à l'ordre chronologique des rêves qui s'y trouvent relatés. Curieux désordre. Serait-ce l'effet d'un simple hasard dû aux aléas de la composition de ce Livre? C'est assez peu probable, quand on sait l'attention que Fellini portait à son 'Librone' et le soin dont témoignent ses éditions. Serait-ce à mettre au compte de la désorganisation dans laquelle notoirement il se plaisait? J'en doute. Car les nombreux reportages qui ont été réalisés sur son travail de cinéaste montrent bien comment en définitive, sous ses dehors brouillons, il dirigeait, contrôlait et ajustait très précisément la réalisation des projets qui lui tenaient à cœur.

Ou peut-on penser que ce diable de Fellini a tout fait pour brouiller les pistes, délibérément? On pourrait le penser, ou le craindre. Car Fellini ne se privait pas de se jouer de ses interlocuteurs et de s'en délivrer en leur servant des répliques qu'il savait brillamment improviser dès lors qu'il se sentait bientôt acculé à avouer quelque vérité qu'il ne voulait pas trop assumer. En témoignent les innombrables interviews où on le voit qui, pas vu, pas pris, s'esquive au moment même où on croyait avoir enfin le fin mot de ce qu'en fait il ne voulait pas dire. ${ }^{23}$

Au demeurant, ne se pourrait-il pas plutôt qu'en l'occurrence il se soit débattu avec lui-même, comme il pouvait, dans la tension où il était entre son engagement dans la réalisation de ses films et les surgissements pour le moins malvenus, en fait durement choquants, qui pouvaient s'imposer à répétition dans ses nuits? Des surgissements, des émergences, des évidences trop brutales, dont il aurait bien voulu ne rien savoir.

Que disent-ils en effet ces rêves et ces réflexions que nous venons de repérer et de remettre, non sans mal, dans leur ordre chronologique? Ils disent une menace d'abord sourde, mais qui bientôt, à proprement parler

${ }^{23} \mathrm{Cf}$. Angeluci (2013), qui était un proche de Fellini et est lui-même cinéaste et écrivain. 
éclate, violemment. La menace de dangers d'abord obscurs, et venant d'en bas, puis, progressivement, celle de méchants chocs, ou d'accidents aéronautiques plus que brutaux, catastrophiques ou du moins désastreux, qu'il vaut mieux fuir, ou dont il vaut mieux, du moins, s'éloigner sans tarder, à toutes jambes.

On a vu que le rêveur, à son réveil, se remémore, observe, dessine et écrit ses rêves, et s'étonne de ce qu'il voit là, sur ce qui se passe là. Parfois même, assez rarement, il faut dire, il en débat. En fait, manifestement, il s'y débat. Il en viendra même, parfois, on l'a vu, à s'interpeller lui-même, à se prendre lui-même à partie, sans ménagement, jusqu'à s'insulter lui-même - se traitant de 'Cretino'-, et jusqu'à se mettre en demeure de prendre acte de ce qu'il a vu et vécu dans la nuit, et, parfois aussi, jusqu'à s'enjoindre de réagir. C'est qu'il pourrait s'agir pour lui de se confronter à ce qui a pu survenir, surgir, dans ses nuits, de si durement insistant, et menaçant, ${ }^{24}$ alors que le Maestro qu'il était derrière la caméra se devait, sous les projecteurs et sous la pression de son entourage et de ses producteurs, de relancer son œuvre, de sorte qu'il lui fallait incessamment jouer et rejouer son rôle de grand ordonnateur d'un cinéma qu'autour de lui on célébrait à l'envi.

\section{RÉPÉTITION ET INSISTANCE INTERPELLATRICE}

Or, toujours à observer ce Livre de près, on a pu voir que ces menaces et désastres aéronautiques, Fellini les connaissait et les vivait de longue date-du moins dans ses nuits. Il les avait remarqués, dessinés et écrits déjà bien avant ces années 1980. Souvenons-nous de ces rêves, que j'ai évoqués plus haut, et qui déjà quelques mois après le début de son analyse avec le Dr Bernhard, en juin 1961, mettaient en scène un avion en difficulté, ou gravement accidenté, et même éclaté. D'un de ces avions, il avait alors écrit qu'il n'arrivait pas à dépasser le sommet de la montagne. 'Il bat péniblement des ailes dans l'air qui devient violet, sans réussir à avancer...' (69). Et en mars 1968, trois ans donc après la fin de son analyse, à la suite de deux rêves successifs eux aussi assez semblables à celui de la planche de Sion, il avait dessiné l'avant d'un avion et écrit en lettres majuscules: 'Mon Dieu! Les pilotes dorment!!'. Et il avait ajouté: '...je m'aperçois avec terreur que l'avion, sorti des nuages qui cachaient

\footnotetext{
${ }^{24}$ On sait l'insistance avec laquelle Jung recourt, dans sa langue, aux termes de 'auseindersetzen', 'Auseinandersetzung', qui parlent, très concrètement, quasi physiquement, de confrontation — des termes que le Dr Bernhard, qui venait d'Allemagne, entendait fort bien.
} 
la vue, est à quelques centimètres du sol. Et s'il y avait des montagnes? Ce serait la catastrophe. Réveille-toi'. Ce qu'il répète, en lettres majuscules: 'Sveglia!' / 'Réveille-toi!' (248-249). ${ }^{25}$

Tandis que le lendemain, à propos d'un autre rêve assez semblable, il avait écrit, sur un mode à la fois descriptif et vivement réflexif, que décidément il lui fallait 'abandonnare l'aereo', 'plonger dans le vide avec son parachute', 'descendre au fond des abysses marins', et enfin, plus impérativement: 'scendere nell'inconscio!' / 'descendre dans l'inconscient!' (514). ${ }^{26}$ Nous sommes alors à la fin mars 1968, presque trois ans après la fin de son analyse avec le Dr Bernhard—et donc après la mort de son analyste - et bien des années avant les rêves du même type, des années 1980, que nous avons vus plus haut.

L'aéronef qui s'écrase sur la page de Sion n'est donc manifestement pas un événement unique dans les rêves de Fellini. Il n'intervient pas là comme un ovni, comme un objet volant non identifié. Il ne s'agit en rien, ni pour Fellini, ni pour nous qui fréquentons son Libro dei sogni, d'un objet jamais vu, non identifiable, et non reconnaissable. Pas plus que la catastrophe qui menace, ou effectivement se produit. Cette scène au contraire est remarquablement récurrente. Insistante. Et pas seulement au début des années 1980. Mais donc déjà dans les années 1960 ou au début des années 1970. Elle est trop insistante pour ne pas s'imposer à l'attention. À notre attention. Et aussi, peut-on penser, et pour autant que faire se peut à celle de Fellini.

Quant à la date à laquelle cette scène de Sion a pu être dessinée, il n'est donc pas impossible qu'elle l'ait été à la fin des années 1960, comme la Fondation Fellini pour le cinéma l'indique dans la récente exposition qu'elle a organisée dans ses locaux de Sion pour marquer l'anniversaire des cent ans de la naissance de Maestro. C'est-à-dire au moment où Fellini réalise I Clowns (1970) - nous aurons donc à nous arrêter à ce film pour apprécier sa place, et peut-être son rôle ou son effet, dans la dynamique des films, et peut-être celle de la vie de notre Maestro. Cette hypothèse concorde avec notre première approche pour dater cette page par l'observation de son format, qui disait qu'elle pourrait bien avoir été dessinée après 1968, peu avant ou peu après la fin de son analyse avec le Dr Bernhard.

\footnotetext{
${ }^{25}$ Nous retrouverons plus loin ce 'Sveglia!'.

${ }^{26}$ Et donc après la mort de son analyste. Notons au passage qu'il est bien rare que ce terme d' 'inconscient' se trouve sous sa plume. Ce terme apparaît d'ailleurs sur cette page avec un point d'exclamation. Il s'agit manifestement ici d'une injonction très concrète et circonstanciée plutôt que d'un recours à un vocabulaire théorique.
} 
Mais il est tout aussi possible qu'elle ait été dessinée et écrite à une date proche du rêve de l'explosion du dirigeable qui dans son Libro dei sogni est daté du 10 décembre $1980 .{ }^{27}$ C'est-à-dire que cette page de Sion pourrait aussi dater de l'année 1980, de décembre 1980, ou qu'elle soit immédiatement postérieure à cette date.

S'il faut trancher entre ces deux hypothèses, je privilégierais plutôt, pour ma part, la seconde, du fait de mon approche à la fois de la tenue formelle et de la thématique de son Libro dei sogni. Car si la composition et le thème du crash d'un avion ou d'un aéronef sont bien représentés à la fois dans les années 1960 et dans les années 1980, ce n'est pas le cas du sauve-qui-peut qu'on voit sur la page de Sion et qu'on ne voit qu'en 1980 dans son Libro dei sogni. Quant à ce que Fellini vit alors, on sait que dans ces années 1980, il vit un nouveau moment de crise qui le fait sérieusement douter de l'avenir du cinéma et de lui-même, ce qui tranche avec les envols - ou essais d'envol—de son imaginaire jusqu'alors. ${ }^{28}$

Mais, en fait, on ne voit pas comment vraiment trancher entre ces deux hypothèses. Alors un doute s'insinue. La question de la datation de ces pages aurait-elle été mal posée? Ou, du moins, demanderait-elle à être revue, reconsidérée ou du moins ajustée? Certes, elle n'a pas été vaine. Elle nous a fait avancer. Puisque nous avons maintenant à considérer deux dates éventuelles. Deux, au moins. Mais on hésite. Et le doute où nous étions, maintenant se fait plus insistant. Ne nous faudrait-t-il pas prendre ici quelque recul? Se pourrait-il que s'ouvre maintenant une autre approche, ou une approche renouvelée, de cette question de la datation de cette page de Sion?

Se pourrait-il que cette scène de crash, ce scénario catastrophe, qui se répète et si manifestement insiste d'une étape à l'autre d'un temps à l'autre de ce Libro dei sogni, perdure et s'impose tout au long de l'œuvre de notre cinéaste et, peut-être aussi, de sa vie? Qu'elle soit comme un thème qui sourdement, obstinément, résonnerait en contrepoint de ses créations, ou, plus exactement, comme une basse continue qui accompagnerait notre Maestro au fil de son œuvre et, peut-être, de sa vie?

\section{LA BASSE CONTINUE ET LE CHANT}

On se plaît à qualifier l'œuvre de Fellini de baroque. Ce qui est un

${ }^{27}$ Ce rêve est daté à tort du 10 janvier 1980 dans le Beaux Arts intitulé Tutto Fellini! (2009: 37).

${ }^{28}$ Pour plus de précisions biographiques à ce propos, cf. Tullio Kezich 2007:chap. 3637. 
peu rapide, et approximatif. Cette qualification a toutefois sa vertu. Elle peut contribuer à renouveler notre approche de la datation de ses rêves. Dans la musique baroque, en effet, on distingue classiquement, si l'on peut dire, la basse continue et les voix de dessus.

À propos de cette musique, on appelle 'basse continue' un motif qui se répète et se relance, parfois presqu'inchangé, et parfois chiffré, tandis que le chant et les autres parties dites de dessus s'élancent, se développent, et se modifient plus librement. Il s'agit assez souvent d'une descente chromatique, assez pathétique. ${ }^{29}$ On parle aussi parfois de 'basso ostinato', de 'basse obstinée', ou de 'groundbass', ou encore de 'basse contrainte', 'soutenue', ou 'obligée'.

Si donc les chocs, entrechocs, brutalités et accidents divers, souvent graves, tout notamment ceux des aéronefs touchés en plein vol et si souvent précipités et écrasés au sol se répètent, obstinément, longtemps presqu'inchangés, dans ce Libro dei sogni et accompagnent si manifestement l'œuvre cinématographique de Fellini et sa vie comme une 'basse continue' et 'obstinée', ne serait-ce pas là le rappel venu de loin, obstiné, continu et presque hors-le-temps, d'une réalité, en l'occurrence la réalité d'une menace qui ne peut pas, ou ne veut pas, se laisser convaincre par les réussites du Maestro, aussi heureuses, brillantes et célébrées soientelles? Il arrive qu'un motif de 'basse obstinée' se retrouve dans la partie haute d'une pièce de musique, où elle prend alors un élan, un essor et connait des développements qui la libère de ses contraintes initiales.

Se pourrait-il que Fellini ait tenté de considérer, de travailler et de reprendre à son compte le motif si brutal et gravement impressionnant des aéronefs menacés et menaçants qui se montre si insistant dans son Libro dei sogni? Qu'il ait tenté de le travailler et de quelque façon de le prendre en main dans son œuvre cinématographique, par les moyens, les pouvoirs et les vertus de son art de cinéaste?

Se pourrait-il que le deuxième dessin de Sion, celui du clown en pleurs, nous aide à mieux comprendre cette tension, si impressionnante, entre cette tonalité de fond pour lui si dure et effrayante du crash des aéronefs et l'œuvre cinématographique si brillante, admirée et admirable de Fellini, et aussi, sait-on jamais, le cours de l'histoire du Maestro?

Voyons cette deuxième page de plus près, en partant, comme pour la

${ }^{29}$ Ainsi en est-il chez Dufay, ou, plus tard, dans les adieux de Didon dans le Didon et Énée de Purcell, par exemple. Ou sous une forme autrement plus ample dans les Variations Goldberg pour clavecin, la Chaconne pour violon seul ou la Passacaille et fugue pour orgue de Bach. Ce qu'on peut retrouver aussi, sous une autre forme, mais dans un esprit assez semblable, dans la musique contemporaine de Webern ou celle de Dutilleux, par exemple. 
première, de l'observation de son format, pour considérer ensuite sa tenue formelle, puis sa thématique.

\section{RETOUR À SION. UN DRÔLE DE CLOWN}

Cette page, tout comme la première, n'est pas datée. Et elle mesure $34,5 \mathrm{~cm}$ sur $32,5 \mathrm{~cm}$, ce qui donne à penser qu'elle a pu être dessinée après la fin de la première partie de ce Livre, soit après 1968, et avant 1982, comme celle du crash de l'avion.

Quant à sa tenue formelle, cette scène nous laisse curieusement en suspens. Comme s'il s'agissait d'un instantané plus proche de la photographie que du cinéma. Même les mots qui viennent pourtant manifestement des personnages qu'on voit là restent suspendus, en points de suspension, comme silencieux - comme était silencieuse l' 'esplosione' que nous avons vue sur le précédent dessin de Sion, une 'esplosione' que Fellini, qui ne craignait pas les oxymores, avait qualifiée de 'silenziosa'. Voilà une scène qui ne fait ni rire, ni sourire. Ni vraiment pleurer, d'ailleurs. Pas plus que la page du crash de l'avion également conservée à Sion. Il est vrai qu'on y voit un corbillard, ce qui, d'ordinaire, n'incite pas trop à une expression très libre des émotions. En donnant le ton de cette scène, de cette page, ce corbillard pourrait bien être au cœur de la thématique de cette page. Je vois d'ailleurs qu'on dit et répète à l'envi qu'il s'agirait ici de 'la mort du clown'.

Il se peut que ce titre, qui dirait de quoi il s'agit, soit justifié. Mais je n'en suis pas trop certain. Il me parait bon, du moins en première approche, que nous nous en tenions aussi longuement que nécessaire à l'expression manifeste de cette scène. Or, en l'occurrence, a-t-on bien vu les traits de couleur du haut de la page et autour du corbillard? Ils sont discrets, mais c'est assez clair: nous sommes dans un cirque, sous le chapiteau et sur la piste d'un cirque. Alors voyons mieux. Deux clowns sont ici mis en scène. Dans la partie haute de la page, sous le chapiteau, on voit un clown à courtes jambes avec cigare et chapeau - une sorte d'Auguste, apparemmentqu'admoneste un personnage dont la silhouette, dans ce Libro, est assez fréquemment celle du Maestro, tandis que dans la partie inférieure de la page, on voit aussi un autre clown, également avec chapeau-plutôt qu'un clown, il semble bien que ce soit ici un Arlequin-, que regarde, de loin, avec recul, une autre représentation assez habituelle de Fellini, flanqué d'un autre personnage tassé sur lui-même, et apparemment accablé.

Un Arlequin? C'est d'autant plus probable que son habit est fait de losanges de tissus de couleurs typique de ce personnage qui nous vient de 
la Commedia dell 'arte, où, assez généralement, il se montre joyeux luron, prêt à jouer mille tours. Mais ici, très manifestement, il éclate en pleurs, tandis que l'éclat de son habit se retrouve, plus obscurément, dans celui de l'Auguste - de cette sorte d'Auguste - qu'on voit en haut de la page pris à partie par le reflet ou l'ombre du Maestro.

Cet Arlequin se penche sur le corbillard, ou il s'y accroche. C'est bien d'un deuil qu'il s'agit. De quel deuil?

\section{DEUILS}

Cette scène n'est pas sans précédent dans ce Libro. Déjà en janvier 1961, donc peu après le début de son analyse avec le Dr Bernhard, Fellini se montre pleurant abondamment sa Giulietta sinon morte, du moins mourante (ill. 6).

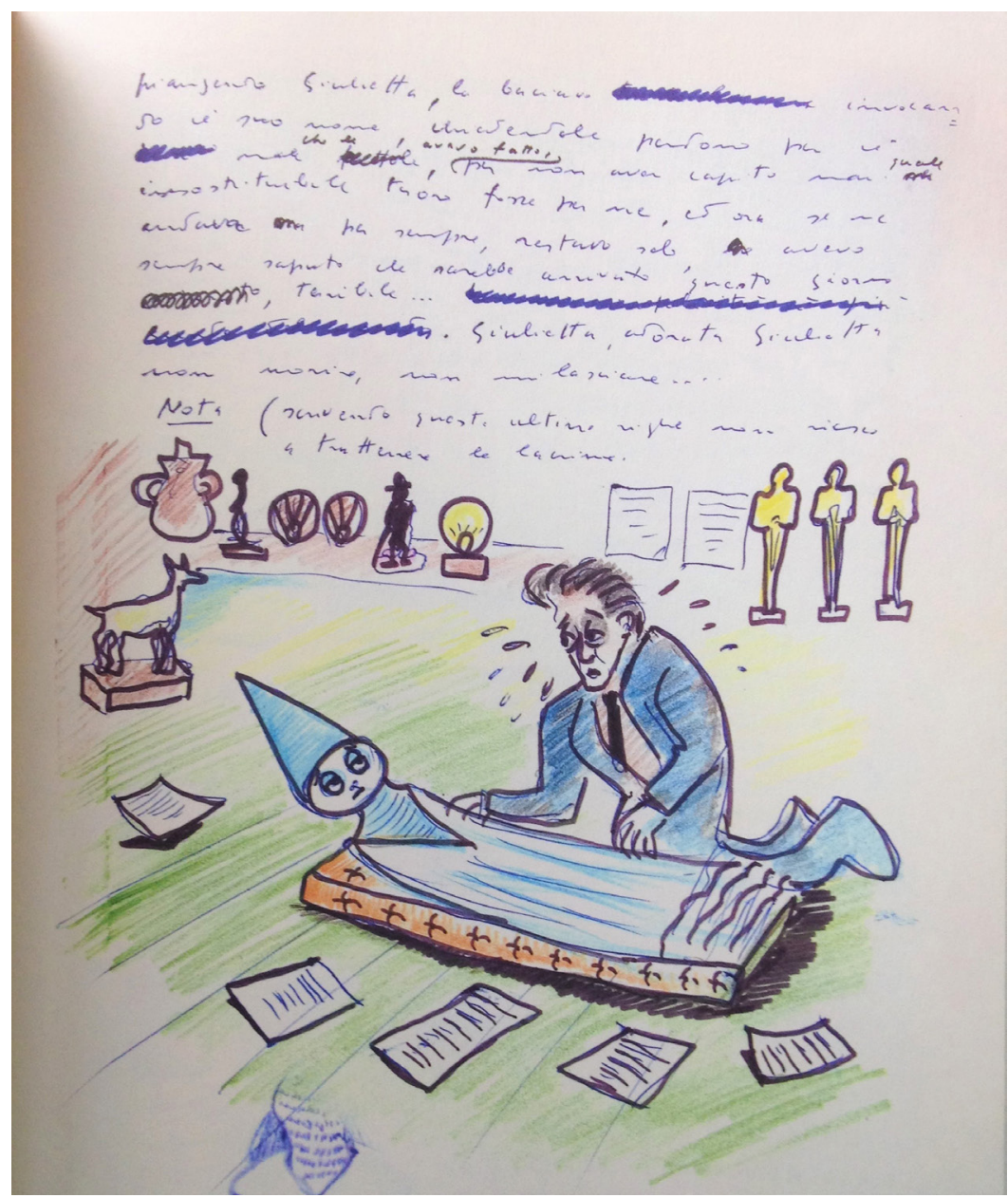

Ill. 6. Page 31 du Libro Courtesy Fondazione Fellini, Rimini. 
Et la même scène de désolation et de deuil éploré se reproduira assez peu de temps après la fin de cette analyse, en janvier 1967 (p. 201). Giulietta est là, mourante, avec ses tresses blondes, dans un cercueil, et lui-même, désespéré, pleure toutes les larmes de son corps. ${ }^{30}$ Remarquons qu'ici il est question de marionnettes, ce que nous retrouverons plus loin. Cette Giulietta mourante porte en effet sur ses tresses blondes 'une couronne de reine des marionnettes'. Les marionnettes, le cirque, la mort viennent de loin dans les rêves de Fellini.

Ce qui pose la question du sort qui dans ce Livre des rêves est fait aux figures récurrentes et obsédantes qui peuplent, animent, et hantent notre Maestro, et aussi son univers cinématographique. Nous retrouvons ici les leçons, qui déjà nous ont accompagnés, de Charles Mauron et de Gaston Bachelard - mais avec cette différence qu'il s'agit ici de la récurrence et du sort d'êtres humains, de figures humaines, plutôt que d'air ou d'eau, ou d'autres éléments naturels. Parmi les personnages qui peuplent ce Livre, la figure de 'Giulietta' est sans doute une des plus présentes et des plus récurrentes. Et aussi une de celles qui évoluent et se transforment le plus manifestement d'une page à l'autre, et d'une année à l'autre.

Je viens d'écrire 'Giulietta' entre guillemets, car, dans ce Livre, elle se présente surtout, jusqu'en 1968 au moins, comme une sorte de Gelsomina, comme une petite personne fragile, mal traitée, déchue, et menacée de mort (p. 47), ou encore comme une figure juvénile, rieuse, infiniment attachante et fragile, presqu'enfantine (p. 62, par exemple). Ces rêves datent des premiers temps de l'analyse de Fellini avec le Dr Bernhard.

Et lorsque Giulietta, dans ces pages, n'est pas 'Giulietta-Gelsomina', mais bien plutôt Giulietta Masina, la vraie Giulietta Masina, sa femme, Fellini la montre comme ramenée sur terre, souffrante, parfois morte. En fait comme la retombée sans illusion de qu'elle était en Gelsomina. Ou alors on la retrouvera en 1965, en bourgeoise romaine assez sotte et assez perdue dans Giulietta degli spiriti, jouant, sans vraiment les déjouer, avec les tours à la fois mondains, passablement ridicules et finalement maléfiques d'une magie pour bourgeois désœuvrés, effrayés et crédules. ${ }^{31}$

Sans pour autant que disparaissent de ces pages, où d'ailleurs elles ne se transforment guère, ces femmes énormément femmes, tout en seins et

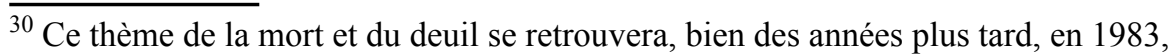
dans $E$ la nave va, un film qui nous intéressera plus loin.

${ }^{31}$ On connait le goût de Fellini pour les voyants et ses fréquentations assez soutenues de magiciens divers, y compris au cours de son analyse avec le Dr Bernhard.
} 
croupe énormes, qu'on se plait à qualifier de 'felliniennes', objets massifs qui l'obsèdent, ou avec lesquelles il voudrait bien jouer, mais qu'il dessine le plus souvent hors de proportion humaine, relevant d'un imaginaire qui oscille entre éblouissement, complaisance et surtout débordement. ${ }^{32}$

Sur cette seconde page de Sion, il s'agit bien d'un deuil. Avec donc la perte, la mort de 'Giulietta' en arrière-plan. Mais ici on hésite à y croire. Le Maestro, qui sur le bord de la page regarde, effrayé, cette scène, y croitil lui-même? On le voit ici qui reste bien en retrait, sur le bord de la page.

\section{Y ALLER ?}

La psychanalyse ou la psychothérapie qu'a vécue Federico Fellini avec le Dr Bernhard de 1960 à 1965 a été un événement important dans sa vie, et elle n'a pas manqué d'effets sur son œuvre. Quand il se rend chez son analyste, Fellini a plus de quarante ans, et il a une belle réputation d'artiste. Avec Lo Sceicco bianco (1952), il avait montré sa maestria attendrie pour dire son goût de cinéaste pour les romans-photos, et aussi pour montrer, déjà, la désolation d'un rêve qui se défait. Avec La Strada (1954), puis dans Le Notte di Cabiria (1957), il avait su voir et faire voir en Giulietta Masina un jeune être infiniment malicieux et touchant, joyeusement aérien, entre fleur et ciel, bien loin de la brutalité massive de l'athlète de foire qu'il sait aussi mettre en scène avec ce qu'il a appris du néoréalisme et de Rossellini. De ses premiers succès de caricaturiste, il avait gardé le sens des portraits, qui dans I Vitelloni (1953) oscille entre la nostalgie de ses années d'adolescence et une charge sans merci contre la médiocrité stupide qu'il avait lui-même bien connue.

Ses films ont créé un cinéma nouveau qui à la fois regarde le mondeil filme alors encore en décors naturels - et dit des moments presque visionnaires qui se concrétisent dans des scènes si fortes en elles-mêmes que de plus en plus, d'un film à l'autre, elles tendent à s'autonomiser et à s'imposer par elles-mêmes, en se présentant, chacune d'elles, comme le condensé ou le précipité visuel d'une nouvelle plutôt que comme les étapes d'un roman au long cours (Gili 2009:chapitre 4).

Dans ces films, la grâce et le drame se côtoient, se regardent, se conjuguent, et nous laissent dans une sorte de mélancolie qui ne perd pas le sourire. Cet acquis du néoréalisme dans une représentation délibérément théâtralisée en même temps que puissamment rêvée étonne ses contemporains, qui parfois hésitent encore à vraiment reconnaitre ses

${ }^{32}$ Qu'on peut voir, par exemple, à la page 125 du Libro-Fellini est alors en analyse depuis plus de trois ans avec le Dr Bernhard. Sur ce thème, cf. Stourzé (2007). 
innovations et sa force créatrice.

Le Dr Bernhard aimait les arts et les artistes. Mais on ne sait pas de quel œil il voyait ce cinéma. Ni ce qu'il a bien pu en dire à Fellini, ou avec lui. ${ }^{33}$ Et moins encore comment il a réagi à la diffusion de La Dolce Vita dès le début des années 1960, au cours donc de l'analyse du cinéaste avec lui. Il serait d'autant plus intéressant de vraiment connaître la façon dont cet analyste a pu voir et recevoir ce film qu'avant sa consécration par la Palme d'or du Festival de Cannes, les réactions qu'il a suscitées ont été plus que vives et contrastées. La polémique a fait rage, l'Osservatore Romano l'a condamné, il a été interdit aux moins de 18 ans, et Fellini en a été lui-même si marqué qu'en avril 1961, il a rêvé qu'il se trouvait violemment pris à partie et admonesté par Mgr Montini, alors cardinal archevêque de Milan, et futur Paul VI (Libro:63).

Si le regard du Dr Bernhard sur le cinéma de Fellini jusqu'au moment de son entrée en analyse avec lui nous intéresserait, il nous serait plus précieux encore de savoir comment il a bien pu accompagner le cinéaste dans la conception et la réalisation de Otto e Mezzo. Ce film est surprenant. Saisissant. L'univers cinématographique de Fellini prend alors une telle distance par rapport à l'art traditionnel de la continuité d'un récit, qu'il s'avère puissamment et terriblement éclaté, presque fait de bric et de broc, en tous cas composé de tant de pièces et de morceaux qu'on est bien en peine de le raconter avec quelque cohérence. ${ }^{34}$ Fellini, dans ce film, et de là pour ses autres créations, travaille de plus en plus en studio-jusqu'à y faire construire la Via Veneto quand il veut en faire un lieu, un objet, de son film.

Fellini vit d'ailleurs de plus en plus mal la conception et la mise en œuvre des films qu'il s'est engagé à réaliser. Surtout quand il aborde un nouveau film à concevoir, et dans les premiers temps d'un tournage. On connait ses atermoiements, détours et ruses en tous genres quand il a à dire quel est son projet, à se rendre sur le plateau, à choisir ses acteurs, à donner un titre au film qu'il va réaliser, ou même qu'il a réalisé. Ainsi en est-il pour le titre de Otto e Mezzo. Les critiques et historiens du cinéma vont d'une hypothèse à l'autre pour expliquer le titre de ce film. Tullio Kezich lui-même se livre à un calcul aussi acrobatique que peu convaincant pour le justifier (2007:240). Alors que ce titre dit ce qu'il en est. Après les films qu'il a réalisés jusqu'alors, celui-ci est une étape, une sorte d'étape, en fait

\footnotetext{
${ }^{33}$ D'ailleurs, malgré les livres maintenant assez nombreux qui se proposent de relater une analyse, qui peut dire ce qui vraiment s'y est joué, et ce qui s'y vit ?

${ }^{34}$ Sur cette organisation en puzzle des scènes felliniennes, cf. aussi l'excellente préface de Italo Calvino à Faire un film (1996).
} 
un demi-pas, vers le cinéma qui se cherche, que Fellini cherche à créer.

Ce que le Maestro vit mal. Il est plus que jamais prêt à se défiler, à se sauver, à se cacher. Alors qu'autour de lui, on l'attend, on le presse, on le flatte, on l'admire, on le célèbre, on se réjouit, on dépense des sommes folles pour la réalisation de ses films, et on l'exploite. Et lui-même jouit de sa maestria quand enfin il s'y met, quand il a tout son monde à sa main, un monde qu'il crée, ajuste et bricole, manipule, déforme, transforme, soumet et plie à ce qui paraît être son bon vouloir ou sa volonté. Fellini, bien sûr, veut aller de l'avant. Il cherche à avancer encore. Mais c'est dur. On le voit tout notamment quand, au milieu des années 1960, au terme donc de son analyse avec le Dr Bernhard, il veut absolument mettre en œuvre un film obsédant, cent fois repensé et remis en chantier, et jamais réalisé, le film qui aurait dû s'appeler Il viaggio di G. Mastorna.

C'est une méchante histoire. Rien moins que l'histoire d'un accident d'avion qui, cette fois, devait passer de ses rêves-cauchemars à un film. Nous avons vu qu'il en a dessiné toute une série dans son Libro dei sogni, notamment après ses nombreux rêves des années 1960 et des années 1980 que nous avons vus plus haut. Cet accident d'avion, ce crash devait conduire à l'au-delà, il devait déboucher sur la vie après la mort. ${ }^{35}$ Sauf que pour concevoir et réaliser ce qui suit cette mort, les différentes versions de son projet se perdent dans les évocations d'une errance vaguement hallucinée de la vie d'ici-bas. Il fait construire à grands frais des décors qui peinent à donner corps à cet au-delà qui lui échappe, ${ }^{36}$ comme lui échappe le héros de cette histoire, dont il veut montrer le désarroi, mais que Marcello Mastroiani lui-même, transformé en l'occurrence en violoncelliste, ne réussit pas à incarner.

Ce projet de film restera dans les limbes où se perd son scénario qui bute à tout instant sur des recherches vaines, des indécisions et contradictions multiples, des obstacles à répétition et d'innombrables appels à l'aide. ${ }^{37}$ Et il hantera Fellini qui ne pourra qu'en reprendre des

\footnotetext{
${ }^{35}$ Dario Zanelli, l'auteur de L'Enfer imaginaire de Federico Fellini (1955), montre que ce film pourrait bien être la réponse, sans Jugement dernier, de Fellini à l'Enfer de Dante qu'on lui aurait trop longtemps infligé pendant ses années d'école à Rimini. Tullio Kezich, de son côté, considère qu'il aurait sa source dans le roman de Dino Buzzati Le Strano viaggio di Domenico Molo publié en feuilleton en 1938 dans le magazine Omnibus (puis en 1942 sous le titre Il sacrilegio dans le recueil I settemessaggeri), alors que Fellini avait 18 ans. C'est en effet à Dino Buzzati que Fellini s'adressera pour écrire le scénario de ce film.

${ }^{36}$ On retrouvera ces décors trois ans plus tard dans les premières scènes de Bloc-Notes d'un cinéaste (1969).

${ }^{37}$ Cf. la Préface de Aldo Tassone à la publication du scénario de ce film jamais réalisé
} 
bribes et des morceaux, parfois des scènes entières, à partir de 1968 dans Toby Dammit, le Satyricon, Roma, E la nave va, et jusque dans La Voce della luna en 1990.

Reste que ce projet de film a buté sur sa réalisation. C'est une butée. Et cette butée est celle de la mort que ce projet veut traverser. Fellini veut forcer le passage. Il veut aller de l'autre côté. Mais en vain.

\section{TOURNER LE DOS?}

Aurions-nous perdu de vue la page de Sion où pleure ce drôle de clown qu'est l'Arlequin accroché à un corbillard? Certes non. C'est l'observation de la tenue formelle de cette page qui nous a conduits jusqu'ici. C'est le suspens où elle est. L'état de suspens où elle nous met. Car nous en sommes aux contradictions et tensions vécues plus que jamais par Federico Fellini-des contradictions et tensions qui certes sont dues assez largement aux pressions à produire que subit le Maestro, mais qui aussi, et plus radicalement, relèvent de la difficulté où il se trouve pour avancer dans son œuvre, pour s'avancer encore. Une autre page de ce Libro peut nous le faire mieux comprendre. On se souvient de la scène où nous l'avons vu fuyant un aéronef qui explose tandis que dans son rêve il s'écriait 'Scappiamo! / Fuyons!'. C'est, nous l'avons vu, à la page 386, où Fellini dit un rêve de décembre 1990 qui le montre, une fois de plus, fuyant le crash d'un avion.

Or sur la page qui fait face à celle-ci, en face donc de ce 'Scappiamo!', se trouve une autre page, un autre rêve, apparemment du même jour, ou de la même nuit. ${ }^{38}$ Dans ce rêve, sur le dessin de ce rêve, on voit qu'est dressé un petit théâtre de Guignols, théâtre de parc ou de jardin, un castelet, d'où sort une bulle, un phylactère, où on peut lire ces mots frappés d'un point d'exclamation, qui semblent bien venir de la bouche du marionnettiste: 'Vuoi tornare a lavorare o no, Buffone' / 'Tu veux retourner travailler ou non, Bouffon'. Et aussi: 'Deve andare avanti!!' / 'Le spectacle doit continuer!!' (ill. 7).

et intitulé Le Voyage de G. Mastorna (Fellini, Buzzati 2013). Curieusement cet auteur et excellent fellinien parle avec enthousiasme du sentiment de 'sérénité' que lui procurerait la lecture de ce projet de film—il est vrai que c'est le ton que Fellini voulait donner à ses dernières séquences.

${ }^{38}$ Dans les conditions ordinaires d'une analyse, c'est évidemment au patient, qu'on appelle aussi, à tort, l'analysé, et qu'il vaut mieux appeler l'analysant, qu'incombe au premier titre cet exercice particulier d'association qu'est la pratique de l' 'amplification' (cf. Gaillard 2001). 


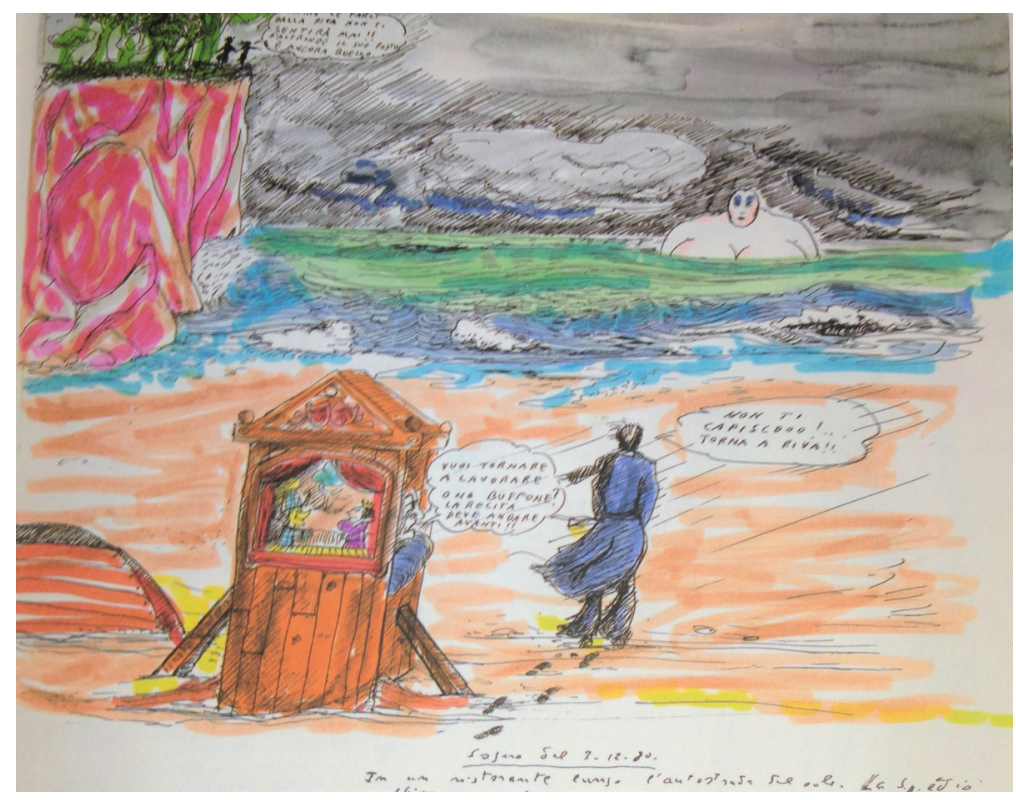

Ill. 7. Page 187 du Libro Courtesy Fondazione Fellini, Rimini.

Cette interpellation se présente comme le rappel d'une règle, d'une sorte d'impératif que connaissent bien les gens du spectacle, du théâtre, du cinéma ou de l'opéra: 'Le spectacle doit continuer'. Quant à ce terme de 'Buffone', il est brutal et sonne ici comme une insulte, ou pour le moins comme une provocation-à moins que ce ne soit un sérieux défi lancé pour obtenir, enfin, une réaction qui tarderait à venir. Cette interpellation provocatrice s'adresse, sur la scène de ce rêve, à un personnage avec écharpe et grand manteau flottant qui ressemble sérieusement à notre Maestro, qu'on voit de dos, qui s'éloigne, s'en va à grands pas pressés et répond, de loin: 'Non ti capisco! ... Torna a riva!!' / 'Je ne te comprends pas! ... Reviens sur la plage!!'.

Ce personnage, que Fellini dessine comme il savait si bien se représenter lui-même, ${ }^{39}$ ne veut décidément rien entendre. Il s'en va au contraire vers la plage, vers la mer, où l'on voit, en perspective, à demi immergée, ou émergente, une de ces femmes à gros seins qui peuplent si manifestement le monde de ses rêves, et largement aussi son cinéma, depuis I Vitelloni au moins. ${ }^{40}$

\footnotetext{
39 J'ai montré au congrès de Rimini (2007) et dans mon article dans les Cahiers Jungiens de Psychanalyse (2009) l'évolution, lente, et finalement la transformation des représentations que Fellini donne de lui-même dans son Libro dei sogni.

${ }^{40}$ Une émergence ou immersion qu'on retrouvera dans son Casanova (1976).
} 
Notre homme va-t-il réagir? Manifestement non. Il ne veut décidément rien entendre. Il tourne le dos à cette interpellation, à ce théâtre de marionnettes et à son marionnettiste. Il n'en veut rien savoir. Or s'agissant de marionnettes et de marionnettiste, il sait pourtant ce qu'il en est. Il a récemment réalisé son Casanova. C'était en 1976. Ce film, pour moi, est vraiment éprouvant. Il est presqu'insupportable. On peut d'ailleurs se demander comment Donald Sutherland a pu supporter de se faire malmener, maltraiter comme il l'a été, véritablement comme une marionnette, y compris physiquement, pour incarner un être aussi détestable, et d'ailleurs ouvertement détesté par Fellini. Au point qu'au risque de tomber dans un jungisme conceptuel trop facile, on en vient à le voir comme une 'ombre' du Maestro. ${ }^{41}$

$\mathrm{Au}$ demeurant, cette page du castelet et celle de ce Fellini qui lui tourne le dos sont de quatre ans postérieures à la réalisation du Casanova. Elles datent de 1980, qui est l'année de La Città delle donne, ce film où Fellini étale, décline et déploie toute une panoplie de femmes énormes, dont on ne sait trop s'il faut décidément s'en garder, ou même les fuir, ou s'il va falloir monter à l'assaut de leurs appâts aussi disproportionnés que des paysages, et dont on ne peut vraiment pas dire qu'ils sont des charmes. En cette fin des années 1970, Fellini explore et expose l'univers terriblement contrasté de ses fantasmes ordinaires. Or qu'est-ce donc qui caractérise la tenue formelle et la thématique de cette deuxième page conservée et exposée à Sion?

C'est, nous l'avons vu, le fait que tout au contraire de se présenter comme une débauche de portraits outrés et d'audaces débridées, cette page dit un moment suspendu, un suspens, un arrêt. Et même un recul, le recul que manifestent les deux personnages, Fellini lui-même et son scénariste, qui se tiennent au bord de la page, à bonne distance du clown-Arlequin accroché au corbillard. Le cadre est celui d'un cirque, mais il s'agit d'un mort, d'un deuil, de la mort. Or c'est en 1970 que Fellini réalise I Clowns. Ce film est un événement dans son œuvre. Il tranche sur le mouvement jusqu'alors constamment et brillamment renouvelé de ses créations, et sur ses œuvres postérieures, dont son Casanova, et sa Città delle donne. ${ }^{42}$

Ce film est dur. Sans facilités ni complaisances. Il ne crée pas un

\footnotetext{
${ }^{41}$ Tandis que Marcello Mastroiani semble vraiment, presqu'à l'évidence-une évidence souvent soulignée — son 'double'. On connait ces photographies de tournage où Fellini impulse, insuffle la position, l'attitude, le geste et la mimique qu'il attend à son acteur, lequel s'y conforme presque exactement (Stourdzé 2009: 115).

42 Pour suivre pas à pas la dynamique de ces films dans leur enchainement et leur contexte culturel en Italie (cf. Manganaro 2009).
} 
monde où on pourrait se lancer et se perdre à corps perdu. Il se présente comme une enquête. Comme une enquête presque post-mortem. La question qui se pose est en effet peut-être nostalgique, mais elle s'avère sans illusion: que sont donc devenus les clowns d'antan? Ils ne sont pas encore morts. Pas tous. Mais ceux qu'on peut encore rencontrer sont des survivants, très âgés, restes épars et assez désolants d'un temps, d'un art, d'un monde d'hier vraiment perdu, et presque perdu de vue.

Ce film est une mise à pied. Pied à terre. Et Fellini se met lui-même dans le champ de sa caméra, participant avec d'autres à cette enquête sur le monde perdu des clowns. ${ }^{43}$ Ce film est d'autant plus dur qu'il se termine sur une impossible ascension ou assomption. L'ascension ou assomption du clown pris dans les lacets, vrais filets et faux effets d'une fête dans lesquels il se trouve pris, tandis que la ronde du cirque dont Fellini a su si bien jouer dans ses films antérieurs devient ici un cortège funèbre.

C'est la fin des clowns. Et, peut-être, la fin, la mort du cirque, de ce cirque de marionnettes que Fellini tentera pourtant de redire, mais sans plus le célébrer, en le représentant au contraire sur un mode on ne peut plus dénonciateur dans son Casanova et dans La Città delle donne.

Fellini, dans ce film de 1970 et dans ceux de la fin des années 70, a su se mettre en question, se mettre à la question, non sans quelque ambiguïté évidemment, mais avec une force et même une violence qui tranchent avec les réussites magistrales qui ont fait son succès jusqu'alors, qu'on célèbre, qu'on ne cesse d'attendre de lui, et dont il a tant joui.

\section{UNE AUTRE INTERPELLATION, ANCIENNE ET RÉPÉTÉE}

On peut se demander, je me suis souvent demandé, ce que Fellini a pu garder en mémoire de ce qui s'est dit lors de son analyse avec le Dr Bernhard. Ou si, comme c'est parfois le cas, surtout quand il s'agit d'enfants ou de jeunes gens, il a avancé ensuite dans sa vie sans plus trop s'en souvenir, ou en tous cas sans trop s'en soucier. À l'invitation de son analyste, il a pourtant pris grand soin de dessiner, d'écrire et de réunir ses rêves dans son Librone tout au long de son analyse et, nous l'avons vu, pendant bien des années ensuite. Il aurait donc pu y retrouver un rêve ancien, du 30 novembre 1960, qu'il avait noté dans les premières pages de son Libro, et qui date des tout premiers temps de son analyse (ill. 8).

${ }^{43} \mathrm{Il}$ avait commencé peu de temps auparavant à se mettre lui-même dans le champ de sa caméra. 


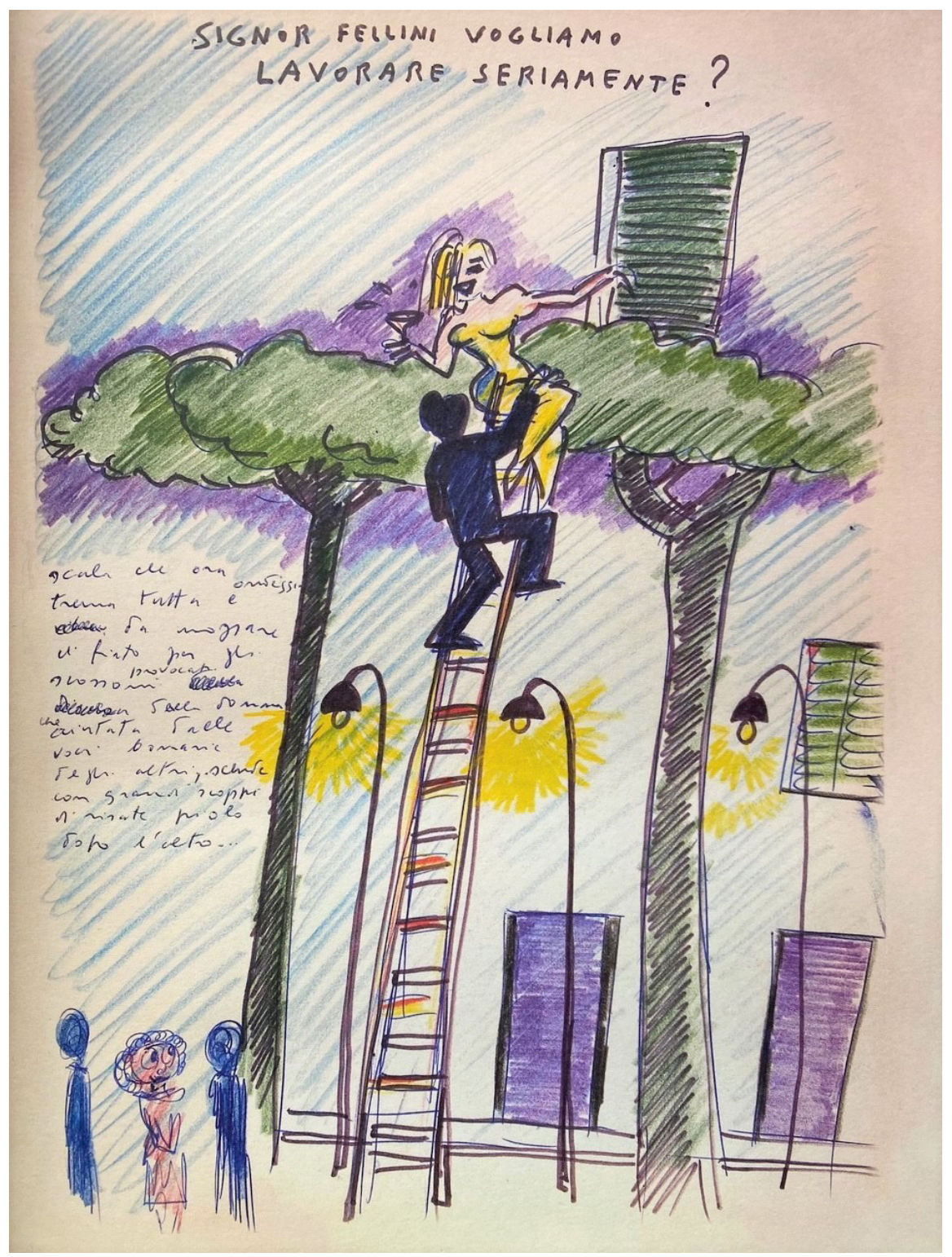

Ill. 8. Page 23 du Libro Courtesy Fondazione Fellini, Rimini.

On le voit là en haut d'une échelle ${ }^{44}$, manifestement intéressé par la jeune femme vers laquelle il tend la main, qu'il voudrait bien rattraper, et attraper. Et on peut lire ces mots, écrits sur la page: 'Signor Fellini, vogliamo lavorare seriamente?' / 'Monsieur Fellini, voulez-vous travailler sérieusement?', ${ }^{45}$

${ }^{44}$ On peut se reporter ici à la scène où il se trouve haut perché sur des échasses (ill. 3). Ces scènes s'inscrivent dans la dialectique et la tension entre hauts et bas dont nous reparlerons plus loin.

${ }^{45}$ La traduction française de Flammarion donne 'voulons-nous travailler 
D'où viennent donc ces mots? Qui donc lui parle ainsi? Serait-ce la jeune femme dont il veut s'approcher et qui ferait mine de le repousser? On ne peut trouver de réponse à cette question sur cette page. On ne peut trouver de réponse qu'en se reportant très loin dans ce Libro, à la page 556. À cette page, en effet, que découvre-t-on? La première partie de ce rêve. Renvoyée dans les dernières pages de ce Livre, dans ses 'Feuilles volantes'. Tout se passant comme si notre rêveur avait voulu, ici encore, brouiller les pistes et nous laisser — ou se laisser lui-même — vraiment loin de ce rêve, de sa portée, et en l'occurrence de cette interpellation pourtant écrite sur la page.

Dans les 'Pages volantes' de ce Livre, à la page 556, on apprend que dans ce rêve, c'est en fait non pas cette jeune personne qui en haut de l'échelle l'interpellerait un verre de champagne à la main. Mais le Dr Bernhard. C'est son analyste qui l'admoneste. Tandis qu'en contrebas, Giulietta aussi l'invite à descendre de cette échelle. Mais le rêveur, qui est donc Fellini lui-même, et la jeune femme en rient, et ne cessent de plaisanter. Tel était donc ce rêve. Mais on ne le découvre pas sans mal. Quel jeu joue donc Federico Fellini, une fois de plus, quand il écrit ce rêve, mais s'arrange pour qu'on ne sache pas trop qui parle? Quand l'origine de ce message qui lui est adressé passe dans les 'Feuilles volantes' de son Librone? Se pourrait-il que lui-même n'en veuille rien savoir ? Qu'il l'écrive, mais ne veuille pas le voir? Il peut être tentant de fermer les yeux. Et de ne rien entendre.

À fréquenter son Libro dei sogni, on se souvient pourtant du très sonore 'Sveglia!' / 'Réveille-toi!', qu'il s'est adressé en 1968 déjà, dans le rêve que nous avons vu plus haut d'un avion qui va s'écraser au sol tandis que les pilotes dorment. Le thème de l'aéronef en détresse, au bord du désastre, est décidément plus qu'insistant dans les rêves du Maestro. Si insistant qu'on va retrouver cette même injonction, ce même 'Sveglia!', bien des pages plus loin dans ce Libro dei sogni, à la page 417, bien des années plus tard, plus de vingt ans plus tard, en $1990 .{ }^{46}$ Fellini mourra peu après, en 1993 (ill. 9).

sérieusement?', une traduction qui a le mérite de rester littérale, mais qui ne tient pas vraiment compte de l'usage ordinaire en italien du 'vous' et du 'nous' dans un tel contexte.

${ }^{46}$ Qui, soit dit au passage, est l'année de La Voce della luna. Je dirai deux mots de ce film plus loin. 


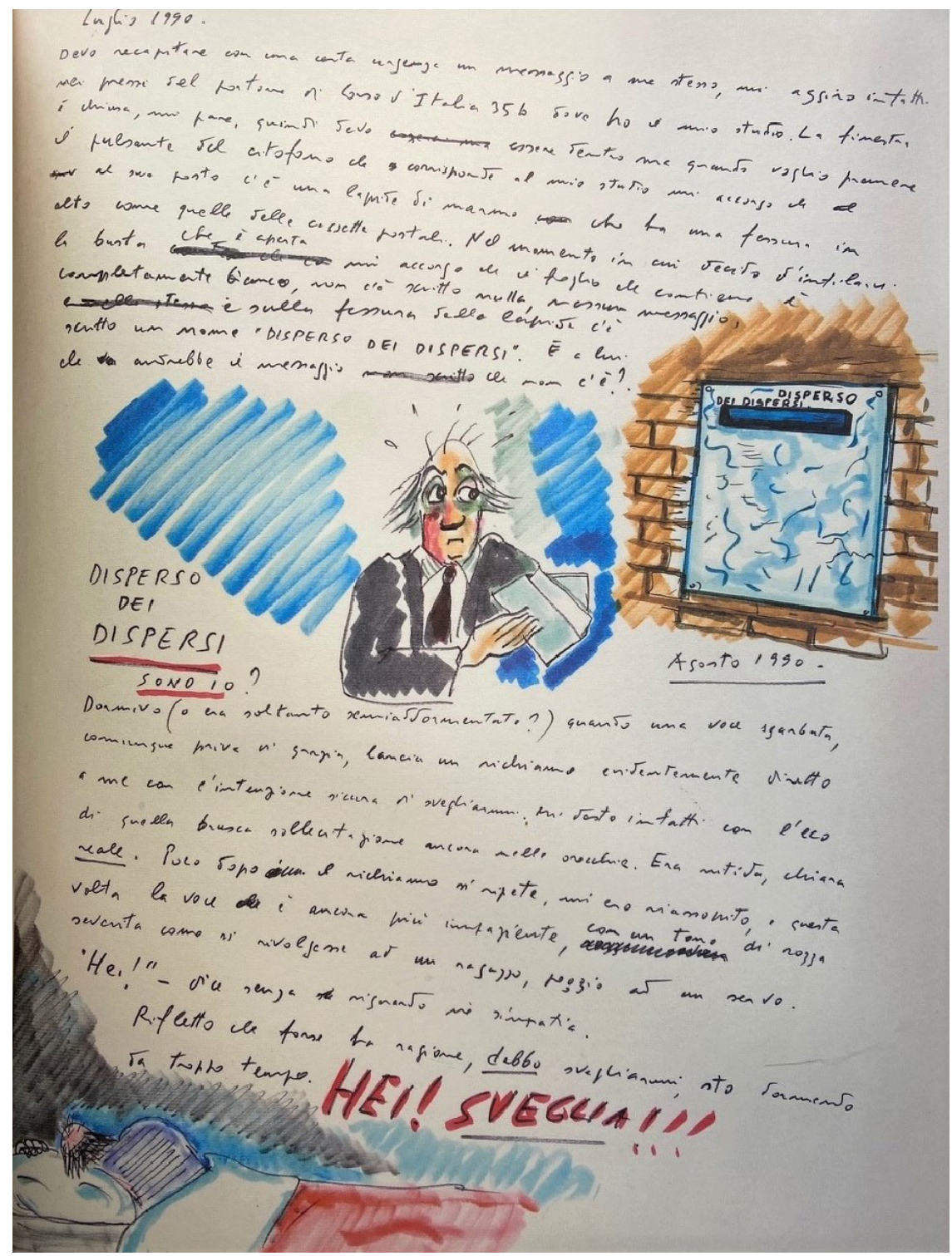

Ill. 9. Page 417 du Libro Courtesy Fondazione Fellini, Rimini.

Cette page, qui est l'une des dernières de ce Livre, est une des plus agitées de l'ensemble. On y voit notre rêveur qui se représente vieilli, à demi chauve, désemparé, au milieu des lignes agitées qui relatent ce rêve. Et ce rêve se termine par un 'Hei! Sveglia!!!' qui répète celui de 1968, sur un mode plus direct et plus rude et brutal encore que ce n'était le cas trente ans plus tôt. Cet appel au réveil, à l'éveil, court ainsi tout au long de ce Livre, des premiers temps de l'analyse du cinéaste avec le Dr Bernhard à ses dernières années. 
À la relation de ce nouveau rêve sur le même motif s'ajoute une réflexion, ce qui reste assez rare dans ce livre et fait écho à celles qu'il avait écrites en 1968. On peut lire: 'debbo svegliarmi, sto dormendo da tropро teтро' / 'je dois me réveiller, je dors depuis trop longtemps'. Sans qu'on sache si cette réflexion a eu lieu dans ce rêve, ou si Fellini se l'est adressée au réveil.

Trente ans ont donc passé entre cette scène et celle de 1968. Le rêveur n'est plus maintenant perché tout en haut d'une échelle, jouant les séducteurs et se jouant de l'injonction de son analyste qu'il renvoie dans les 'Feuilles volantes' de son Librone. Il se voit et se montre maintenant de face, de front, et ne cache plus son désarroi, un désarroi plus radical encore qu'en 1968, qui frôle l'affolement, tandis qu'il veut s'adresser à lui-même un message dont il note que la feuille qui le porte 'est complètement blanche, qu'il n'y a rien d'écrit dessus...' (référence). La scène reste en suspens, en définitive aussi muette que le message qu'il veut se faire parvenir.

Voilà qui nous ramène à la scène du clown en pleurs. Regardons mieux encore cette page de Sion, que nous cherchons à dater.

\section{LA MORT DU CLOWN?}

Voyons, à côté de ce clown en pleurs de cette page, un personnage apparemment accablé qui s'exclame 'molto commovente'. Ce personnage qui se tient bien à distance de l'Arlequin qui pleure, c'est Zapponi, Fellini écrit son nom sur la page. Bernardino Zapponi est scénariste. Et c'est un ami du couple Federico-Giulietta Masina. Il collabore avec Fellini depuis 1968. Voilà donc qui confirme que cette page est postérieure à 1968. Mais comment préciser à quelle date postérieure à celle-là elle a pu être dessinée?

Ces thèmes de la mort et du passage à un autre monde, hantent, on l'a vu, notre cinéaste depuis déjà le milieu des années 1960. Et ces thèmes se retrouvent dans l'une et l'autre de ces deux pages de Sion, dans le crash de l'avion et dans la scène du clown en pleurs accroché au corbillard-ce qui les apparente, et pourrait bien nous amener à penser que décidément la date de cette scène du clown qui pleure ne sera pas plus simple à établir que celle du crash de l'avion. Sauf qu'un autre dessin, une autre scène du Libro va nous aider à avancer.

En 1966, alors donc que l'analyse de Fellini avec le Dr Bernhard vient de se terminer par la mort de son analyste, qu'il se débat avec le projet de Voyage de Mastorna et que déjà il veut l'abandonner, voici que 
dans un de ses rêves une main lui tend des feuilles encore blanches. Et derrière cette main qui se tend, on voit, son dessin nous le montre, un dessin, un portrait, non pas du Dr Bernhard, mais de Carl Gustav Jung, le mentor de son analyste (ill. 10).

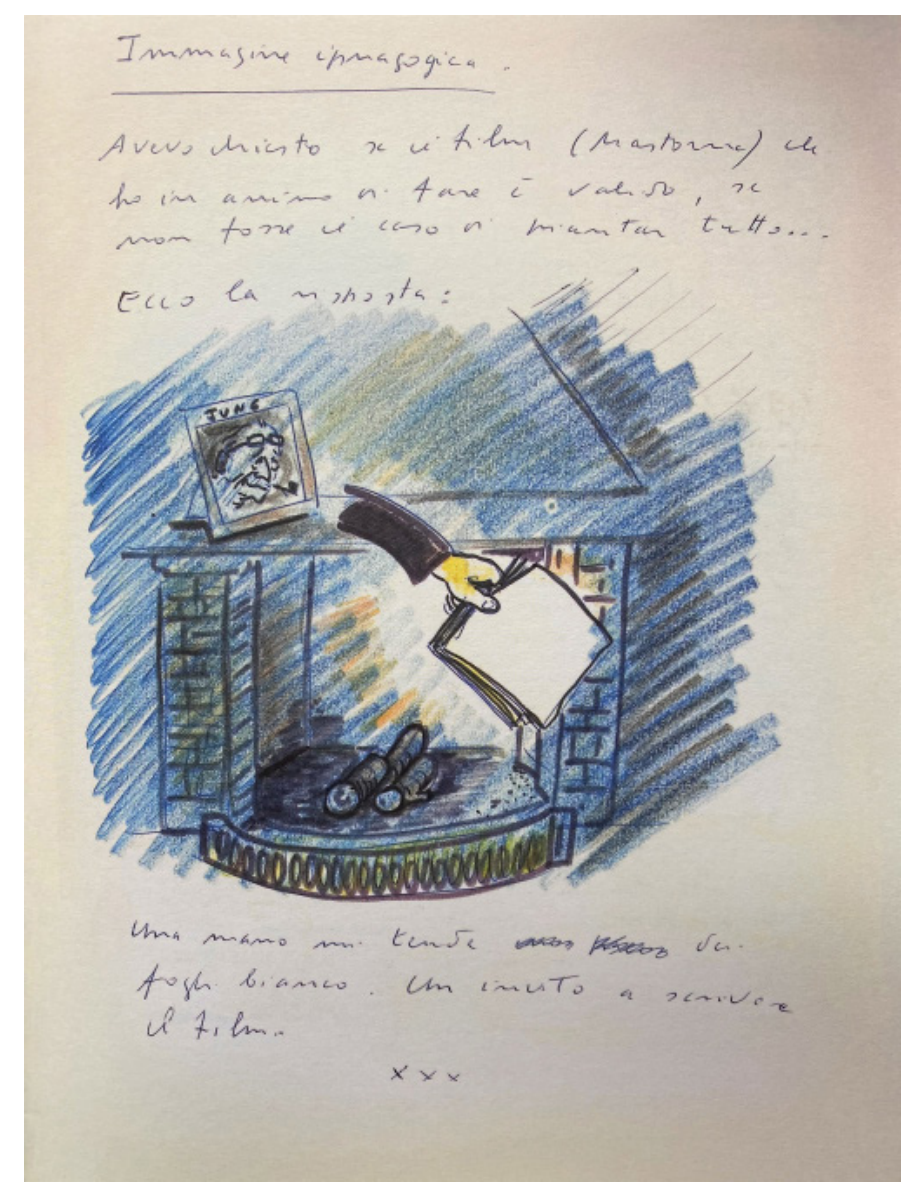

Ill. 10. Page 165 du Libro Courtesy Fondazione Fellini, Rimini.

Fellini lisait volontiers Jung. Plutôt dans le désordre, évidemment. Je ne sais donc pas s'il a jamais lu l'essai de Jung sur Picasso, et moins encore s'il en a jamais parlé lors de ses séances avec le Dr Bernhard ${ }^{47}$ C'est un fait en tous cas que dans cet essai assez sombre du début des années trente, Jung s'interroge sur le mauvais pas où se trouve Picasso à cette époque de sa vie et de son œuvre (Hillman and Shamdasani 2013; Gaillard 2013,

\footnotetext{
${ }^{47}$ Fellini partageait avec George Simenon une admiration constamment réaffirmée pour Jung (Fellini, Simenon 1998).
} 
2016). Il s'en inquiète, et tente en particulier de comprendre la présence alors insistante dans cette peinture des gens du cirque, en particulier celle des clowns et celle d'Arlequin. ${ }^{48}$ On remarque que le clown de cette planche de Sion porte un habit d'Arlequin, fait de pièces et de morceaux. Et on remarque aussi que c'est également le cas de cette sorte d'Auguste qu'on voit en haut de cette page. ${ }^{49}$

Si Fellini a lu Jung, il sait de quelle menace de mise en morceaux, de quel morcellement, il peut s'agir. ${ }^{50}$ Et s'il ne l'a pas lu, il peut le savoir, par son expérience propre, par sa pratique du dessin, par la composition de ses films, surtout à partir de 1969-1970, et par le cours de sa vie. Mine de rien, le tragi-comique de cette scène du clown en pleurs touche Fellini au plus vif de ses angoisses à la fois de crash, d'effondrement, d'éclatement et de mort. Sous ses grands airs de magicien-cinéaste, il est hanté par un sacrifice impossible - le sacrifice des figures idéales de son imaginaire, de lui-même en Maestro, et de sa lanterne magique qui pourrait bien, sait-on jamais, voler en éclats.

Quant à la date de cette planche, on aurait pu penser qu'elle est contemporaine du dessin de 'Giulietta' mourante, et qu'elle aurait donc été réalisée au début des années soixante, dans les premiers temps de l'analyse de Fellini avec le Dr Bernhard. Mais on a appris que Zapponi ne travaillait pas encore avec lui. Il nous a donc fallu relancer et réajuster notre enquête pour en arriver à donner une date à cette page où pleure l'Arlequin.

On avait aussi pu penser que cette scène pourrait bien être contemporaine de la première planche de Sion, celle de l'avion qui s'écrase, et qu'elle pourrait dater du début des années 1980, c'est-à-dire du début de la dernière décennie de la vie de Fellini, une décennie, la dernière de sa vie, qui sera pour lui méchamment dure et sombre, tant pour ce qui est de sa vie quotidienne que pour ce qui concerne ses capacités de création. Mais si la tenue formelle et le thème de ce dessin permet cette hypothèse, rappelons-nous ses dimensions et sa présentation presque carrée-ce qui, quand on les rapporte à celles des pages du Libro, ne permet que difficilement de la soutenir.

\footnotetext{
${ }^{48}$ Il dit cette inquiétude de bien des façons, en particulier en évoquant à demi-mot la figure du danseur de corde telle qu'elle apparaît dans le Zarathoustra de Nietzsche, et le sort tragique qui le menace.

49 À propos du Clown blanc et de l'Auguste, cf. Merlino (2007), le chapitre intitulé 'Les ambassadeurs de ma vocation', et Gili (2009: 53).

${ }^{50}$ Jung, à partir de sa fréquentation de l'iconographie et de la littérature des alchimistes, et à partir de sa pratique de clinicien, parle dans sa langue de 'Zerstükelung', ce qu'on traduit ordinairement par 'morcellement', alors qu'à mon sens parler de 'mise ne morceaux' serait plus juste (cf. Gaillard 2017:chapitre VII, 1978:38 sq et 151 sq).
} 
J'en conclus que cette planche du clown en pleurs a été plus probablement réalisée au cours des années dont ce Libro, curieusement, ne nous dit rien, entre 1969 et 1973, et plus précisément en 1970, au moment donc où Fellini concevait ou réalisait I Clowns, son film sans doute le plus autobiographique, écrit Jean Gili, mais sans trop s'en expliquer (référence). Dans ce film, Fellini se filme lui-même. Il est le cinéaste, et il est dans le champ de sa caméra, ce qui est nouveau. Il se filme lui-même ou se fait filmer parmi les acteurs de son film. On le voit là non pas en grand ordonnateur perché sur la haute machine de tournage qui porte sa caméra, mais au sol, parmi les autres. Il est lui-même un des protagonistes de la recherche et de la rencontre des vieux clowns qui ne le sont plus. Et ce film le regarde.

\section{DRÔLE DE CONFRONTATION}

Avec ce film, Fellini se confronte lui-même à la fin de ses tours et détours de magicien du cinéma. Il est alors bien près de les mettre en question, d'en faire son deuil peut-être. De se mettre sérieusement en question. On le voit sur la partie haute de cette page qui admoneste, qui rappelle à l'ordre l'Auguste, lequel, d'ailleurs, avec le gros cigare qu'il arbore ostensiblement et sa mine fermée ne semble pas vraiment disposé à l'entendre. On sait que Fellini se plaisait à classer tout un chacun dans la catégorie des Augustes ou des Clown Blancs. ${ }^{51}$ On ne s'étonnera pas trop de voir qu'il se voyait lui-même en Auguste - et on notera qu'il mettait aussi Jung dans cette catégorie, tandis que pour lui Freud était un Clown blanc.

Avec ce dessin, on reste dans le cadre d'un cirque. Et cet Auguste reste entouré d'ombres fantomatiques. Dans la partie basse de cette page, tout tourne autour d'un corbillard. Il s'agit bien de la mort. D'un mort. Mais qui donc est mort en l'affaire? Regardons mieux encore. Le clown en pleurs s'écrie: 'mon père... hei! Père... hei!!', en français sur la page. ${ }^{52}$ On dit et répète qu'il s'agirait ici de la mort du clown. Mais alors à qui donc s'adresserait cet appel au bord du corbillard? Et si c'était non pas cet Arlequin, mais le père et créateur des clowns qui était mort? Si c'était le cinéaste passé maître dans la création de tant de figures clownesques poussées jusqu'à cet extrême où le plus singulier rejoint le plus typique?

\footnotetext{
${ }^{51}$ Cf. Merlino (2007) dans le chapitre intitulé 'Les ambassadeurs de ma vocation', et Gili (2009: 53).

${ }^{52}$ I Clowns a été tourné en grande partie à Paris.
} 
Quand il relate son rêve de 1970, il est tout près de se le dire, d'en prendre acte, ou du moins il tente de le faire, lui, le père de tant de clowns et de clowneries. Il tente d'en prendre acte dans ce rêve du corbillard, dans sa transcription de ce rêve, et dans le film qu'alors il conçoit ou déjà réalise sur le monde perdu des clowns.

Mais avec un écart entre ce rêve et ce film. Le rêve est plus direct et plus radical. Ce rêve lui parle de sa propre mort-même si on reste sur la scène d'un cirque. Dans son film, certes il se fait voir, et nous fait voir, un corbillard et l'impossible ascension ou assomption du clown sur fond de funérailles qui se voudraient festives. Mais lui-même, dans ce film, reste un des protagonistes de cette scène de deuil, et il est derrière la caméra, et par là, de fait, ce n'est pas lui qu'on enterre. Le cinéma engage, plus que le rêve, à une prise de distance et à une élaboration secondaire plus contrôlée. ${ }^{53}$

Il reste, dans ce film, sur le bord de ce que pourtant il veut voir, et savoir. Alors que sur la page de Sion, il s'agit bien, expressément, de la mort du père des clowns, même s'il se garde bien de vraiment le dire, de se le dire peut-être, se tenant comme il se tient sur le bord de la page, sans vraiment tourner le dos à ce corbillard, mais à bonne distance, et en compagnie de son scénariste qui, lui aussi, se garde bien de trop s'émouvoir et de prendre l'événement au sérieux. En cette année 1970, avec ce dessin et avec ce film, ce diable de Federico Fellini s'approche plus que jamais de ce qui le regarde, mais qu'il se garde de bien de voir trop directement, et trop sérieusement.

Il faudra bien des années, près de 12 ans, pour qu'à la suite d'un autre rêve dont nous avons parlé plus haut (cf. sous-partie 'L'art de brouiller les pistes'), il s'adresse à lui-même en écrivant, en février 1982, cette fois vraiment sans ambages ni faux fuyant: '...scomparso, il re del cinema' / '...il a disparu, le roi du cinéma', '...sicuramente debellato, Fellini' 54 / '...il est vaincu à coup sûr, Fellini', 'Insomma, ero morto' / 'Bref, j'étais mort'.

Serait-il donc vraiment mort, Federico Fellini? Certes, le Maestro souffre. Son rapport à lui-même, à son corps, à son âge, à son temps n'est vraiment plus ce qu'il était. Il s'en plaindra, amèrement, au cours des années 1980, ses dernières années, malgré les honneurs qui s'accumulent et le témoignage d'admiration dont il pourrait jouir.

${ }^{53}$ Par élaboration secondaire on entend après Freud ce travail qui consiste à rendre plus acceptable intellectuellement ou émotionnellement un fait trop difficile à recevoir et à considérer.

54 'Debellato' est un terme très fort (cf. note de bas de page 21). 
Ces années 1980 seront celles, pourtant, de E la nave va et de $L a$ Voce delle luna, des films qui pour moi comptent parmi les plus accomplis, les plus forts et les plus justes de son œuvre. Le dirait-il lui-même? Il lui faudrait alors - on le lui souhaiterait - distinguer le sacrifice, apparemment nécessaire, de ses complaisances dans l'imaginaire et la puissance symbolique, irremplaçable, de ce qu'il peut et sait créer.

\section{POUR CONCLURE. ENTRE HAUT SET BAS}

Dater ces pages n'a pas été simple.

La première de ces pages, celle du crash de l'avion, qui parle d'une menace d'éclatement, de chute, d'effondrement, et de fuite éperdue, relève d'une thématique qui traverse l'œuvre et la vie de Fellini. Elle peut avoir été dessinée et écrite à l'occasion de diverses étapes de sa vie et de son œuvre, de la fin des années 1960 à la fin des années 1970.

La seconde, celle du corbillard sur fond de cirque, date, quant à elle, très vraisemblablement de 1970 , du moment où il a réalisé $I$ Clowns, le film où il est sans doute le plus proche de lui-même à la fois dans ses rêves, dans son cinéma, et de la reconnaissance des forces et des formes qui l'habitent, de ce qu'il peut en faire, et de ce qu'il pourrait devenir.

Ces deux pages peuvent avoir été contemporaines, mais elles ne le sont pas nécessairement. Elles s'inscrivent l'une et l'autre dans une ligne de fond qui perdure, persiste, et insiste, tout au long de la vie et de l'œuvre de Fellini, dans cette ligne de fond que j'ai pu qualifier de 'basse obstinée', ou de 'basse continue'. Mais avec une sérieuse différence de centre de gravité entre l'une et l'autre. Le crash de l'avion vient des airs et fait s'enfuir à toutes jambes les pauvres humains que ce crash peut détruire, alors que la scène du clown qui pleure a vraiment lieu à terre-même s'il faut remarquer que nous sommes là encore sur le terrain, sur la piste, d'un cirque.

L'année 1970, celle de I Clowns, est un des moments les plus durs et les plus cruciaux de l'œuvre et de la vie de Fellini. Il tombe alors de haut. Le temps des jeux et artifices dans lesquels il a si longtemps montré sa maestria est alors sérieusement dépassé-avant qu'il ne retrouve un souffle autrement créatif, toujours rêveur, mais bien différemment, avec Roma (1972), Amarcord (1793), et La Voce della luna (1990), tandis qu'avec son Casanova (1976), Prova d'orchestra (1979), La Città delle donne (1980) et Intervista (1987), il met son art au service d'un regard sans merci sur les rêves et mirages qu'il s'est plu à cultiver jusqu'alors (ill. 11). 


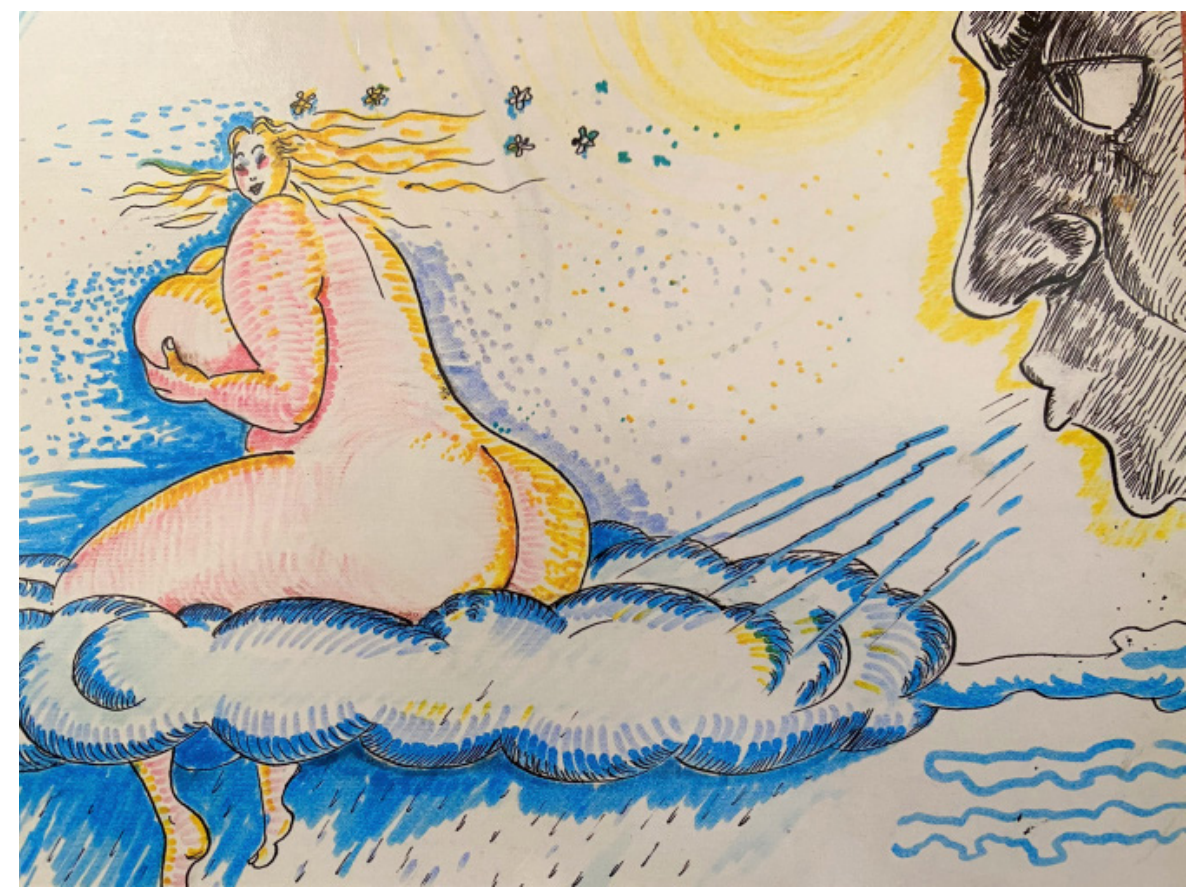

Ill. 11. Page 305 du Libro Courtesy Fondazione Fellini, Rimini.

Cette page du Libro date d'avril 1975. Donc des derniers mois de l'analyse de Fellini avec le Dr Bernhard. Il s'agit d'une 'image hypnagogique', précise Fellini. C'est-à-dire qu'elle lui est venue dans une sorte de 'rêve éveillé', un exercice de l'imagination plus proche de l'éveil que ne le sont d'ordinaire les rêves de la nuit. Je suppose que le Dr Bernhard n'en a pas été mécontent—et je me demande bien ce qu'il a pu en dire. ${ }^{55}$

Le rêveur dans cette scène se montre lui-même éloignant avec force et vigueur, et d'un souffle et d'un air furieux, l'image si insistante d'une de ces femmes tout en seins et en fesses dont il se plait tant à cultiver l'image, et qui là s'en va sur un nuage — comme un nuage. On peut se demander si Fellini s'en est vraiment souvenu au cours des décennies suivantes.

Avecl'œuvre deFellini, son univers etsa vie, on a affaireà des avancées qui relèvent de rythmes différents. À ses réussites cinématographiques plus que magistrales qui ne cessent de nous impressionner et de nous éblouir, et de le réjouir lui-même, répondent tant bien que mal les expressions d'un

\footnotetext{
${ }^{55}$ A propos d'éveil, qu'on revoie le 'Sveglia' (1968: 20) ci-dessus, qui se retrouvera bien plus loin dans ce Libro, et dans sa vie, à l'occasion d'un rêve de juillet 1990 (ill. 9 ci-dessus), dans un contexte de grand désordre et désarroi.
} 
mouvement de fond qui avance comme il peut, et s'avère parfois durement éprouvant.

Ce mouvement de fond, cet accompagnement de fond, tout au contraire d'aller toujours de l'avant, d'être toujours in progress, obéit à une temporalité qui lui est propre. ${ }^{56}$ Non pas qu'il soit hors-le-temps - 'zeitlos', comme Freud pouvait le dire de l'inconscient. Mais il a sa propre persévérance. Et ses propres moyens d'insistance. On a vu en effet que le thème des aéronefs qui explosent et s'écrasent, qui est celui de la première page de Sion, peut se répéter, ou donner lieu à des transformations plus ou moins notables, mais qui en fait le laissent assez semblable à luimême. On peut avoir l'impression que ce thème, ce motif de la catastrophe aérienne, refuse l'avancée, qu'il est décidément réfractaire au changement, qu'il renâcle obstinément devant la transformation.

Sauf qu'à force d'insistance, cette 'basse obstinée' ou 'continue' finit parfois par se faire entendre, par se placer, et même par s'imposer, de sorte qu'alors elle entre dans le champ, dans le champ de la conscience, même si elle peut s'avérer vraiment malvenue, plus qu'importune, et si dérangeante qu'on ne peut vraiment lui faire place. C'est l'histoire de l'impossible projet de $\mathrm{I} l$ viaggio di G. Mastorna.

L'évidence du corbillard et du clown en pleurs sur la deuxième page de Sion parle, quant à elle, de cette butée si difficile à ignorer et à considérer qu'est la mort. Quelle mort? Qu'est-ce donc qui peut, ou doit, ou devrait mourir? Notre Maestro peut tenter de prendre de la distance, il peut vouloir s'en distancier, jusqu'à presque tourner le dos à ce qui se présente là, et s'impose. Serait-ce lui-même, ce lui-même qu'il s'est construit qui se trouverait là mis en cause, et mis à mal, sérieusement menacé?

L'œuvre cinématographique de Fellini tente de reprendre à son compte, et de prendre au sérieux ce qui a commencé à s'exprimer, à se donner à voir, à reconnaitre et à vivre dans ses rêves. Le thème de la mort et du deuil se retrouvera au cœur de E la nave va, ce film si impressionnant dont j'ai évoqué le ton à la fois éprouvant, émouvant et caricatural.

D'une étape à l'autre de son Libro dei sogni et aussi de son œuvre, ce thème des aéronefs qui explosent et celui des clowns en pleurs progressivement s'esquissent, s'insinuent, parfois s'esquivent, mais aussi insistent, résistent, et s'affirment avec une insistance obstinée qui peut certes tourner à la répétition et dans ce cas, parfois, ils tournent en

${ }^{56} \mathrm{~S}$ 'agissant des processus de création, deux livres restent particulièrement éclairants, L'Ordre caché de l'art, de Anton Ehrenzweig (1974), et Le Corps de l'œuvre, de Didier Anzieu (1981). 
rond, mais ils n'en tentent pas moins d'intervenir, d'interpeller l'œuvre et l'homme, jusqu'à s'imposer parfois-à défaut de se faire vraiment entendre.

C'est que notre Maestro avance et se débat comme il peut. On connait ces photographies de lui en cinéaste-cameraman et ces plans de certains de ses films, dont Fellini Roma, où haut perché sur les machines portant sa caméra et le porte-voix à la main il se fait allègrement et puissamment aérien, grand ordonnateur du monde des acteurs, figurants et techniciens qu'il dirige et qu'il a à sa main.

Ces hauteurs ne sont pas sans danger. Les aéronefs ont leurs faiblesses. Ils sont vulnérables. Ils peuvent même éclater. Comment alors éviter la catastrophe? Il vaut mieux s'enfuir, et au plus vite, on l'a vu. Ou déjouer l'événement. Par le jeu. Le jeu du cirque. Un jeu qui peut faire rire. Mais qui a sa gravité. Celle des clowns.

On peut aussi s'en réjouir, plus ingénument. C'est le sourire si attachant de Gelsomina. Qui est grimé, il est vrai. Mais qui enchante. Sauf qu'il va changer. Sous le sourire qu'on veut croire si heureux de Giulietta Masina, le portrait, d'un film à l'autre, se fait moins confiant, et moins crédule, au point d'en devenir presque caricatural quand on regarde Giulietta degli spiriti et La Città delle donne. C'est qu'avec la femme, les femmes, on ne sait trop comment s'y prendre. On est vite débordé.

Fellini, dans ses rêves, regarde, et se regarde. On aurait aimé, bien sûr, qu'au-delà, ou plutôt à l'occasion de leur transcription dessinée et écrite il s'étonne de ce qui se présente et se représente là. Qu'il discute ce qu'il se fait voir dans ses nuits. Et nous fait voir et vivre dans ses films. On aurait aimé qu'il en débatte avec lui-même. Comme Jung l'a fait dans son Livre Rouge. Débattre peut conduire à se battre. À affronter ce qui se présente. À s'y confronter.

Dans les rêves de Fellini, et dans son œuvre, il s'agit d'une affaire en cours. Dont il est partie prenante. On a vu, j'espère, ou du moins entrevu au fil de ce que j'ai pu dire de ses rêves, comment il est passé, au rythme des transformations de son cinéma, du haut des airs et des échasses et échelles auxquelles où il a pu s'accrocher à de bien étranges et inquiétants monstres marins, qui, en contrebas hélas échouent sur la plage, comme dans Otto e mezzo, ou s'imposent sur sa pellicule, et sur la plage (ill. 12). 


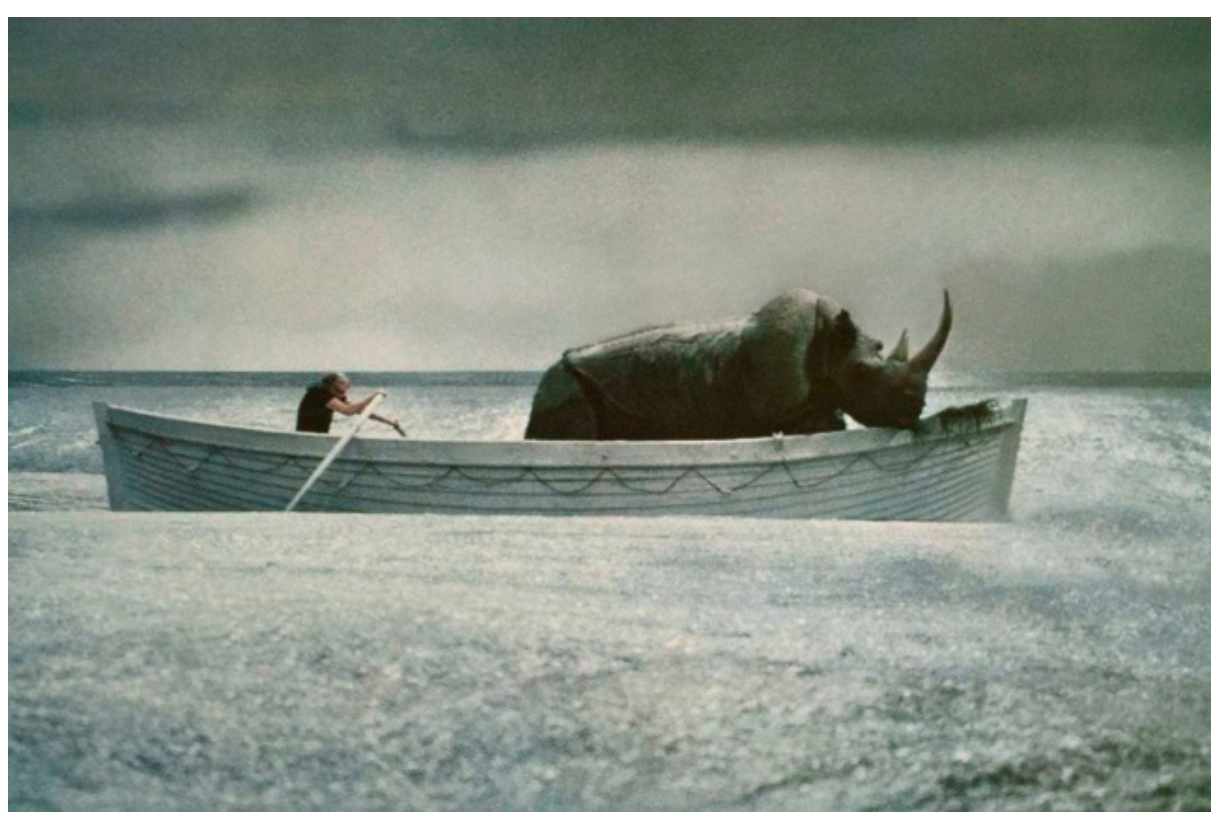

Ill. 12 le rhinocéros sur le bateau de E la nave va. (Gili:88).

Cette scène se trouve dans les dernières séquences de $E$ la nave va. Ce film de 1983 est pour moi un des plus excellemment felliniens et des plus impressionnants de son œuvre cinématographique. ${ }^{57}$ Fellini y met magistralement en scène son univers le plus rêvé avec la diva admirée et décédée dont on va jeter les cendres à la mer et toute une brochette de personnages d'un temps passé à la fois dérisoires, dépassés et touchants ${ }^{58}$, que rattrapent l'actualité et les brutalités d'un abordage qu'ils n'ont pu concevoir ni prévoir. Le film se termine sur le transport maritime de ce rhinocéros aussi disproportionné que nourricier. ${ }^{59}$

On a qualifié ces scènes de fins de films de surréalistes. À tort. Elles sont subréelles et puissamment archaïques. Elles relèvent de ce travail de fond dont j'ai dit qu'il le hantait, et qui tente, mais longuement en vain, de faire surface et de se laisser apprivoiser.

Le centre de gravité et la tonalité de ce Libro dei sogni, et aussi de l'œuvre cinématographique de Fellini ont bien changé d'une œuvre à

\footnotetext{
${ }^{57}$ Stéphane Marti et Nicolas Rouiller ont conçu et réalisé une belle installation sur le thème de ce film pour l'exposition Fellini organisée en l'honneur de l'anniversaire des cent ans de la naissance de Fellini par la Fondation Fellini pour le cinéma à la Maison du Diable à Sion, en 2020.

${ }^{58}$ On y voit au passage Pina Bausch, qui traverse la scène et cette étrange compagnie, ce qui contribue à la profondeur douloureuse de ce film.

${ }^{59}$ Aimé Agnel a mis en évidence ces fins de films dans son article intitulé 'Jung et Fellini. L'inconscient crée des images, le film reste à faire' (2012).
} 
l'autre, et d'une décennie à l'autre de sa vie. Cette gravité était déjà bien là dans son œuvre, dès La Strada, et elle a traversé bien des jeux qui n'étaient pas que des effets de cirque. Elle s'est nouée dans I Clowns, jusqu'à ce qu'enfin, dans La Voce della luna, il se mette et nous laisse le dos bien à terre et le regard tourné vers les lumières de la nuit, dans une conscience alors d'autant plus juste et plus interrogative qu'elle s'est faite lunaire (Stein 1993).

Suivre d'une page à l'autre ce Libro dei sogni, c'est accompagner pas à pas ce cinéaste admirablement créateur qui apprend chemin faisant les aléas, les émergences et les découvertes toujours surprenantes et parfois sérieusement dérangeantes d'un rapport à l'inconscient qui s'avère à la fois défensif, nourricier et fécond. La création en provient. Et de là l'artiste procède comme il peut, à la manière qui est la sienne, et avec les moyens dont il sait se doter.

\section{BIBLIOGRAPHIE}

Agnel, Aimé. 2012. 'Jung et Fellini. L'inconscient crée des images, le film reste à faire'. Cahiers jungiens de psychanalyse 135:7-17.

Angeluci, Gianfranco. 2013. Segreti e bugie di Federico Fellini. Cosenza: Luigi Pellegrini Editore.

Anzieu, Didier. 1981. Le corps de l'œuvre. Paris: Gallimard.

Bachelard, Gaston. 1941. L’Air et les songes.Paris: José Corti.

Calvino, Italo. 1996. Faire un film. Paris: Le Seuil.

Bellocchio, Lella Ravasi. 2009. L'Inconscio creatore. Bergamo: Moretti e Vitali.

Buzzati, Dino (1942). I sette messaggeri. Milano: Arnoldo Mondadori Editore.

Ehrenzweig, Anton. 1974 [1964]. L'Ordre caché de l'art: essai sur le psychologie de l'imagination artistique. Paris: Gallimard, traduit de l'anglais par Francine Lacoue-Labarthe et Claire Nancy.

Fellini, Federico. 2007. Il Libro dei miei sogni. Mostra a cura di Tullio Kezich e Vittorio Boarini; con la collaborazione di Giuseppe Ricci. Milano: Rizzoli. 
Fellini, Federico et Georges Simenon. 1998. Carissimo Simenon. Mon cher Fellini. Paris: Cahiers du Cinéma.

Fellini, Federico et Damian Pettigrew. 1994. Fellini, je suis un grand menteur. Paris: L'Arche.

Fellini, Federico, Dino Buzzati, et Brunello Rondi. 2013. Le Voyage de G. Mastorna. Préface d'Aldo Tassone. Paris: Sonatine éditions.

Gaillard, Christian. 1978. Musée imaginaire de C. G. Jung. Paris: Stock.

-2001. 'Amplification et pensée après Jung.' Topique. Revue freudienne 76:73-86.

— 2006. 'The Arts'. In Renos Papadopoulos (éd.) The Handbook of Jungian Psychology. London, New York: Routledge:324-377.

- 2007. 'Dire et non-dit du rêve.' Federico Fellini. Il Libro dei miei sogni. Convegno Internazionale Presieduto da Tullio Kezich. Rimini, Teatro degli Atti, 9-10 novembre. Rimini: Fondazione Federico Fellini:107-131.

. 2009a. 'Dire et non-dit du rêve. Le Libro dei sogni de Federico Fellini.' Cahiers jungiens de psychanalyse 129, juin:47-81.

- 2009b. 'Le Livre des rêves. Fantasmes, angoisses et obsessions du maestro.' Tutto Fellini ! Exposition au Jeu de Paume. Rétrospective à la Cinémathèque française. Paris: Beaux Arts éditions.

2009b. 'Dire e non detto nel sogno. Il Libro dei sogni di Federico Fellini.' In Christian Gaillard, Lella Revasi Bellochio L'inconscio creatore. Attorno al Libro dei sogni di Federico Fellini. Bergamo: Moretti \&Vitali:9-63.

- 2010.'Takingwing and the ordeal of immersion: Reflections on Federico Fellini's Il Libro dei sogni.' Jung Journal. Culture and Psyche 4 (2), Spring:31-61.

. 2013. 'Jung, Picasso et le bleu.'Revue de psychologie analytique 1, juillet:33-73.

2016. 'L'Originaire est quotidien.' In Sacco François \& Éric

Robert (éd.) L'Origine des représentations. Regards croisés sur l'art préhistorique. Paris: Ithaque:81-93.

- 2017.Jung. Paris, PUF.

Gili, Jean A. 2009. Fellini. Le Magicien du réel. Paris: Gallimard. 
Hillman, James and Sonu Shamdasani. 2013. Lament of the Dead. Psychology after Jung's Red Book. New York: Norton \& Company.

Kezich, Tullio. 2007. Federico Fellini: sa vie et ses films. Paris: Gallimard.

Manganaro, Jean-Paul. 2009. Federico Fellini Romance. Paris: Gallimard, POL.

Mauron, Charles. 1963. Métaphores obsédantes et mythe personnel. Paris: José Corti,

Merlino, Benito. 2007. Fellini. Paris: Gallimard.

Risset, Jacqueline. 2007. 'L'ombelico del sogno. Il metodo di Federico Fellini.' Federico Fellini. Il Libro dei miei sogni. Convegno Internazionale Presieduto da Tullio Kezich. Rimini, Teatro degli Atti, 9-10 novembre. Rimini: Fondazione Federico Fellini:231-243.

Stein, Murray. 1993. Solar conscience. Lunar Conscience. Wilmette: Chiron.

Stourdzé, Sam. 2007. 'Fellini, le faiseur d'images.' Federico Fellini. Il Libro dei miei sogni. Convegno Internazionale Presieduto da Tullio Kezich. Rimini, Teatro degli Atti, 9-10 novembre. Rimini: Fondazione Federico Fellini:57-78.

2009. Fellini. La Grande Parade. Paris: Anabet/Le Jeu de Paume.

Zanelli, Dario. 1955. L'Enfer imaginaire de Federico Fellini. Rimini: Guaraldi.

\section{FILMOGRAPHIE}

Fellini, Federico. 1963. 81/2. Roma: Cineriz

. 1965. Giulietta degli spiriti. Rome, Paris: Federiz, Francoriz Production.

- 1968. Toby Dammit. Rome, Paris: Produzioni Europee Associate, Les Films Marceau-Cocinor.

- 1969. Fellini Satyricon, Roma, Alberto Grimaldi.

. 1969. Fellini: ADirector's Notebook. New York: NBC Productions Int'1.

1970. I clowns. Roma, Paris, Grünwald: Rai, ORTF, Bavaria Film, Compagnia Leona Cinematografica.

1972. Roma. Roma, Paris: Ultra Film, Les Productions Artistes 
Associés.

— 1973. Amarcord. Roma: F.C. Produzioni, P.E.C.F.

- 1976. Il Casanova di Federico Fellini. Roma: PEA.

- 1979. Prova d'orchestra. Roma: RAI-TV, Daimo Roma, Albatros Monaco

-1980. La città delle donne. Paris: Gaumont.

- 1983. E la nave va. Roma, Paris: RAI, Vides Cinematografica, Gaumont. UNO.

1987. Intervista. Ibrahim Moussa, Aljosha Productions, RAI-

1990. La voce della luna. Roma: Mario e Vittorio Cecchi Gori. RAI-UNO.

Morin, Gérald. 2013. Sur les traces de Fellini. Paris: Fox Pathé Europa.

Pour les illustrations 1 et 2, courtesy Fondation Fellini pour le cinéma, Sion.

Pour les illustrations 3 à 11, courtesy Fondazione Fellini, Rimini.

Pour l'illustration 12, courtesy Vides produzione/Rai Uno/Collection Christophel/ArenaPAL. 